\title{
Overview on the Blockchain-Based Supply Chain Systematics and Their Scalability Tools
}

\author{
Houssein Hellani ${ }^{1,2 *}$, Layth Sliman ${ }^{2}$, Abed Ellatif Samhat ${ }^{3}$, Ernesto Exposito ${ }^{1}$ \\ ${ }^{1}$ Université de Pau et des Pays de l'Adour, E2S UPPA, LIUPPA, Anglet, France
}

${ }^{2}$ EFREI Engineering School-Paris, Villejuif, France

${ }^{3}$ Lebanese University, Faculty of Engineering-CRSI, Univ. Campus, Hadat, Lebanon

\begin{abstract}
Modern IT technologies shaped the shift in economic models with many advantages on cost, optimization, and time to market. This economic shift has increased the need for transparency and traceability in supply chain platforms to achieve trust among partners. Distributed ledger technology (DLT) is proposed to enable supply chains systems with trust requirements. In this paper, we investigate the existing DLT-based supply chain projects to show their technical part and limitations and extract the tools and techniques used to avoid the DLT scalability issue. We then set the requirements for a typical DLT-based supply chain in this context. The analyses are based on the scalability metrics such as computing, data storage, and transaction fees that fit the typical supply chain system. This paper highlights the effects of Blockchain techniques on scalability and their incorporation in supply chains systems. It also presents other existing solutions that can be applied to the supply chain. The investigation shows the necessity of having such tools in supply chains and developing them to achieve an efficient and scalable system. The paper calls for further scalability enhancements throughout introducing new tools and/or reutilize the current ones.
\end{abstract}

Keywords:

Internet of Things (IoT);

Distributed Ledger Technology (DLT);

Supply Chain;

IOTA; Scalability;

Blockchain as a Service (BaaS)

Article History:

Received: 16 May 2021

Revised: 14 July 2021

Accepted: 27 July 2021

Published: 23 August 2021

\section{1- Introduction}

Product complexity and market globalization have led most companies to collaborate for the purpose of providing the best quality and prices. Apparently, a successful collaboration requires a reliable, effective, and error-free supply chain system. Each step of the production process ranging from the reception of the source material, industrialization, and finally to transmitting the product to the consumer, establishes the supply chain. At any stage of a supply chain, collaboration is essential among partners that end up with the final product tagged by all stakeholders: producers, vendors, retailers, consumers, and any involved party [1] for a successful end-to-end production. Furthermore, the supply chain consists of a combination of independent partners where each partner represents a centralized standalone system, leading to a data transparency problem, which is a significant issue related to the supply chain. Research studies [2-4] show that distrust between the partners of a supply chain is a critical issue that hinders collaboration $[5,6]$.

Furthermore, today, the clients demand more detailed information regarding the goods, including quality of service, manufacturing source, safety, conformity documents, etc. However, traditional supply chain platforms suffer from many drawbacks that hinder effective collaboration among stakeholders. This is due to data partition, which is often distributed independently in different stakeholders' databases without any global governance and control. Thus, achieving trust among partners has to be done through empowering visibility and transparency along the supply chain so that the

* CONTACT: hussein.hellani@hotmail.com

DOI: http://dx.doi.org/10.28991/esj-2021-SP1-04

(C) 2020 by the authors. Licensee ESJ, Italy. This is an open access article under the terms and conditions of the Creative Commons Attribution (CC-BY) license (https://creativecommons.org/licenses/by/4.0/). 
involved parties can track their goods back to their source. Traceability can be achieved Using Internet of Things (IoT) technology.

IoT devices have recently become essential elements in the supply chain [7, 8]. IoT technology can deliver the collected records from all the networks to improve productivity and traceability of the supply chain. Distributing many IoT devices along the supply chain allows stakeholders to monitor their products' progress instantly and precisely. According to the Cisco report [9], Evidently, the increasing number and diversity of IoT devices will increase supply chain complexities [10, 12]. Furthermore, further data charges will encircle the supply chain where the exchange of such data leads to data consistency and platform scalability problems. As a result, current supply chain schemes do not satisfy most business requirements [20].

Blockchain is a good candidate that controls data systems end-to-end along the supply chain [13]. It forces trust among partners and enables full transparency of the data records throughout a supply chain system underlaid by a cryptographicbased platform [14]. In addition, the DLT relies on a shared distributed ledger among participants, which has significant improvements over trust and collaboration. Furthermore, Blockchain resists changing or removing any records without keeping marks. Partners, therefore, have precisely the exact copy of the ledger data and thus have a granular vision over whole network details. Many studies $[15,17]$ survey the implementation of Blockchain and its critical aspects, and different solutions mention that it is a suitable instrument for supply chains to overcome the trust and collaboration issues. Blockchain is called the 'truth machine' [18] that prevents users/companies from deception. Moreover, several pilots and Proofs-Of-Concept (POC) schemes have been established in recent years using a Blockchain on top of the supply chain for traceability and transparency purposes [17]. However, Blockchain may suffer from scalability and performance issues in an active supply chain environment [19].

During the supply chain's development and its recent integration with the Blockchain, the supply chain experiences decentralized difficulties dissimilar to the traditional centralized ones. These new challenges are common to all business sectors. Our goal is to shed light on the scalability concern as it is one of the major challenges that could hinder the overall new DLT-based supply chains. This paper considers the traditional and DLT-based supply chain scalability challenges and progress. Several proposals and projects target the Blockchain installation on top of their supply chain systems to benefit from the outstanding features and fix the current issues. Since Blockchain is not scalable enough and may hinder the overall processes, some of these projects include additional tools to mitigate scalability gaps. The core of this study revolves around these tools and their impact on supply chain scalability. The following can summarize the paper contribution:

- Highlight the challenges and requirements of the current and DLT-based supply chains in terms of scalability;

- Disclose the technical sides of the current supply chain projects based on DLT and expose their scalability tools;

- Analyze the overall tools and their employments to verify their scalability impacts on the supply chain;

- Shed light on the different solutions for further testing and developments to achieve high scalability.

The rest of this paper is arranged as follows: The methodology of this paper is presented in section 2, section 3 presents the current supply chain challenges and investigates the challenges/benefits of applying DLT on the supply chain and the requirements of the typical supply chain platform. Section 4 lists the main tools of different projects that would lead to the future proposal(s). Section 5 surveys existing DLT-Based Supply Chain Solutions. Section 6 analyzes the overall plans and tools to verify the possibilities to reach the predefined efficient situation, and we conclude in section 7.

\section{2- Research Methodology}

This paper aims at illustrating the evolution process of the traditional centralized system into modern decentralized ones. It investigates the challenges the traditional supply chain encounters and elucidates the Blockchain intervention in solving these issues. The study presents a considerable number of DLT-based projects, reveals their structure, and sheds light on their different tools that tackle the scalability issue of Blockchain. The study is limited to exploring the top available tools that enhance scalability in the supply chains. The criteria for which these tools are selected are based on their roles in advancing scalability directly or indirectly. Thus, the search keywords are Blockchain-based supply chain, modern supply chain, IoT-enabled supply chain, scalability solutions, and DLT limitations. In the discussion, we address the presented questions in this work by discussing the tools' influences on each case's scalability. The analyses are based on the data storage, computing, and transaction fees criteria of the typical supply chain system, listed in the requirements section. The novelty of this study is that it explores the current projects and embodies their technical part in new figures inspired by their whitepapers. Its novelty is also presented in extracting the Blockchain tools of the existing DLT-based solutions that aim to adjust the supply chain development and enhance scalability. It is presented and elaborated in a manner that helps the researchers and developers adopt the convenient supply chain platform concerning their utility. Figure 1 illustrates the methodology research of this study. 


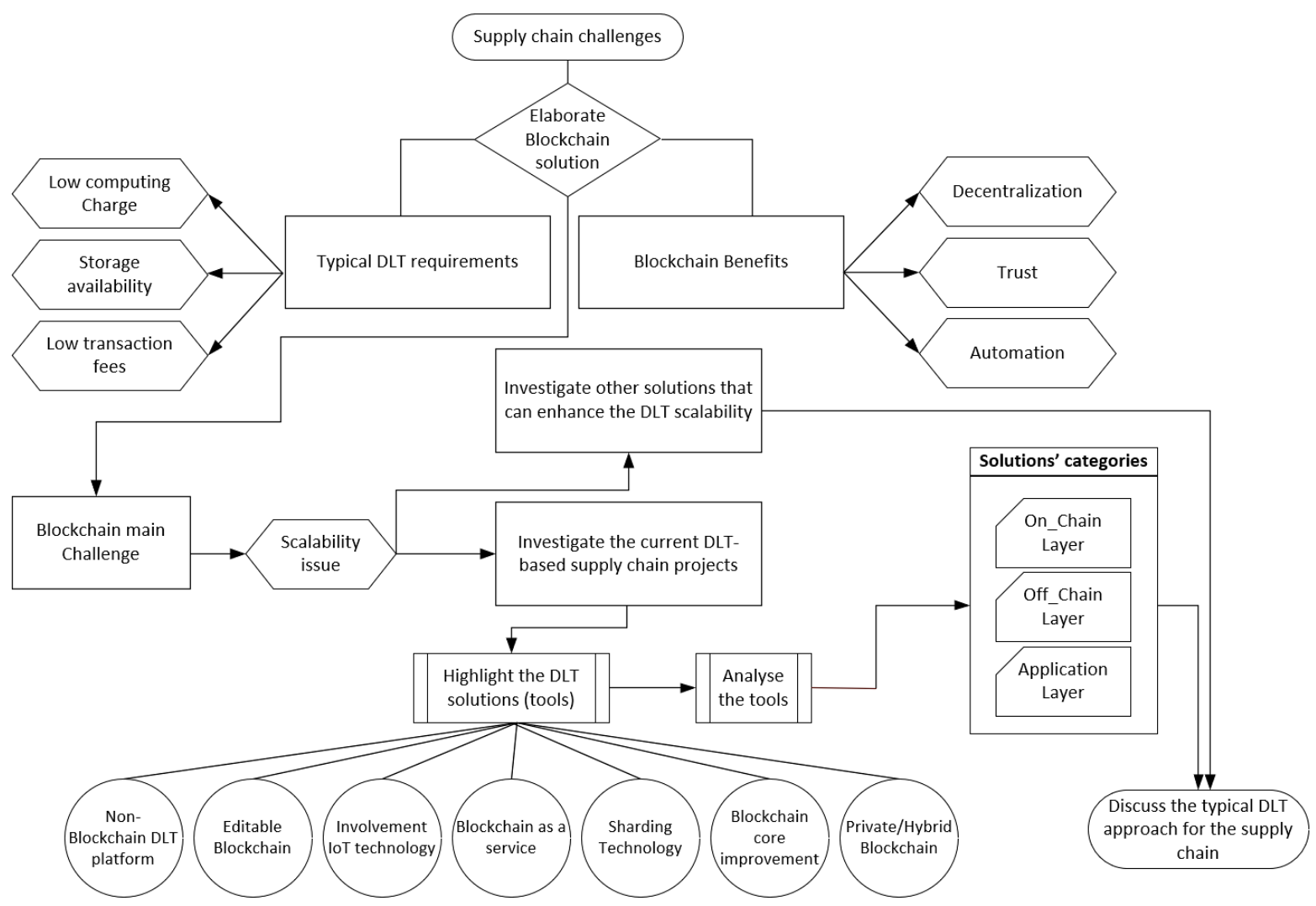

Figure 1. Research Methodology.

The following questions summarize the research methodology of this paper:

Q1. What are the challenges of current supply chain systems and the benefits/challenges behind integrating Blockchain with them?

Q2. What are the existing DLT-based supply chain projects? Structures and directions?

Q3. What are the tools that could be used to boost scalability within the supply chains?

Q4. What are the other DLT tools/solutions that can benefit supply chain scalability if applied?

Q5. What are the suggestions to achieve typical DLT-based supply chain platforms?

By addressing these questions, we conduct two types of research, obtaining a literature review. To perform this study, we searched in the first type for scientific databases papers hosted in Google Scholar, IEEE, ResearchGate, ACM, etc. In the second type, we browsed several whitepapers and blogs on sites related to certain researchers who have conducted studies on the same topic. DLT technology is a relatively new subject adopted by supply chains, and this novel subject has a limited number of reviews [30].

Table 1. Existing surveys on DLT-based supply chain.

\begin{tabular}{|c|c|}
\hline Surveys & Roles \\
\hline $\begin{array}{l}\text { Wang et al. (2019) and Al-Jaroodi \& } \\
\text { Mohamed (2019) }[17,21]\end{array}$ & Reviews industrial applications across different domains. \\
\hline $\begin{array}{l}\text { Hald et al. (2019) and Wang et al. } \\
\text { (2019) }[22,23]\end{array}$ & $\begin{array}{l}\text { Surveys the enabling and constraining roles of the technology from a business/ management- } \\
\text { oriented perspective. }\end{array}$ \\
\hline $\begin{array}{l}\text { Pournader et al. (2020) and Azzi et al. } \\
(2019)[24,25]\end{array}$ & Organizes the theoretical implications of adopting Blockchain in supply chains. \\
\hline Scully and H“obig (2019) [26] & Analyzes the impact of DLT on different supply chain flows through case studies. \\
\hline Tribis et al. (2018) [27] & $\begin{array}{l}\text { Offers a systematic mapping study focusing on the research aspect of Blockchains, recognizes } \\
\text { challenges that remain unsolved. }\end{array}$ \\
\hline Helo and Hao (2019) [28] & Conducts a brief literature review to introduce Blockchain technology and utilization. \\
\hline Saberi et al. (2019) [29] & $\begin{array}{l}\text { Adopts DLT in several organizations and hosts in their work summary statistics useful in } \\
\text { benchmarking the current practice. }\end{array}$ \\
\hline Gonczol et al. (2020) [30] & Evaluates the applicability of Blockchains in the supply chain domain. \\
\hline Yadav, Jyoti, and Ranjana Shevkar [31] & $\begin{array}{l}\text { Categorizes the different scalability solutions into different layers, including on-chain and off- } \\
\text { chain, and compares their impacts. }\end{array}$ \\
\hline
\end{tabular}


Table 1 displays the current surveys that tackle this subject entirely or partially. Consequently, this paper considers the innovative supply chain projects and presents them in detail. Considering the projects that indistinctly display their technical details related to the Blockchain mechanism, the paper focuses on evident industrial projects that apparently provide the required technical information. This paper can help the researchers building their future supply chain projects. It is considered a guide because it analyzes and explains the innovative tools required to ensure supply chain scalability.

\section{3- Supply Chain Challenges}

\section{3-1- Traditional supply chain challenges}

The supply chain experiences enormous variations over time, with high demand on integrating it with IoT technology. Consequently, the current supply chain infrastructure investments surcharge the traditional systems with high loads, leading to information loss [19]. The current supply chain infrastructure constitutes multi-centralized-based organizations where each represents an independent platform that belongs to one or more supply chains. These systems depend profoundly on standalone, often different, and centralized platforms [16]. The group of databases involved in the production are autonomous, heterogeneous, and distributed [32]. Therefore, the inflexibility in interchanging data among these databases is referred to as the hard-coded nature of different data form standards. Two obvious examples are Walmart and Cisco [33]. The collaboration would be limited due to the organizations' desire to use their platforms and control their data. They use different protocols and workarounds to enable interoperability to a particular supply chain. XML, ebXMB, and UDDI are some workarounds used to facilitate interoperability, but they are ineffective in heterogeneous databases due to different standards of databases. File transfer that uses fix format such as XQuery is the only protocol used to interchange data between organizations [32]. The uncontrolled informational data of the centralized systems causes outsize counterfeit, huge information loss, and bad business reputation.

The widespread IoT adoption is triggering profound changes in global manufacturing [34]. Basically, the IoT systems are heterogeneous and categorized under various administrative areas [35]. Three types of IoT technology can be used unitedly or independently within the supply chain: things-oriented that provide sensing ability, semantic-oriented that enables accessing knowledge, and internet-oriented that acts as middleware [10]. Mainly, the industrial field has five IoT techniques [36]: WSN, RFID, cloud computing, middleware, and IoT software. Using these techniques, IoT assists producers in collecting data precisely, such as senses temperature disparity, calculate the color degree and the elapsed time compared to human capabilities [37]. Although IoT technology facilitates the production progress, helping the fabrication part, and providing high control, it charges peripheral devices and servers with high data load [36]. The current network infrastructure fails to employ the IoT's full potential and manage the enormous received data within such centralized situations [19]. In that way, a significant IoT power is dismissed. Unfortunately, centralized solutions fail to manage the enormous incoming data and control them as they were designed [19]. Nowadays, there are no reliable frameworks or infrastructures intended to connect the massive heterogeneous and disparate IoT devices and their connected services, not to mention data analysis and aggregation [38]. The current standalone supply chain systems are ineffectively struggling to provide some of the requirements in the means of trusted third parties and workarounds [19].

Besides, scalability is the primary concern referring to the distributed nature of partners. A product that passes across several geographical areas includes many critical documents such as invoices, ISO certificates, letters, proofs, customs, etc., that require different levels of communications between partners. A study displayed that two hundred connections are needed to realize a single product delivery [39]. On a large scale, traditional systems suffer from high traffic connections. Unfortunately, the current solutions do not guarantee safety, integrity, and in-time delivery of goods/documents, which leads to data loss and confusion. Furthermore, the current systems are vulnerable to exploitation and attack on a large scale due to their widespread and lack of control [16].

\section{3-2-DLT-Based Supply Chain}

\section{3-2-1- Benefits}

Blockchain is an efficient candidate used to address the above supply chain issues [40]. It is a peer-to-peer (P2P) fully decentralized system that provides trust among non-trusted partners [41]. The Blockchains have essential characteristics: tamper resistance, tamper evidence, shared decentralization, distributed trust, multiple-party consensus, independent validation, and record-keeping immutability [42]. Mainly, Blockchain has three implementation types: Public Blockchain (permissionless) is accessible for everyone, such as Ethereum. Private Blockchain (permissionned) is limited to one enterprise such as Hyperledger, and consortium Blockchain, which is limited to a network of determined companies. The Blockchain aids the supply chain in managing its limitations and improving its functionalities through the below features:

- Decentralization: Blockchain is not controlled by a central authority but by the entire network of members and establishes the rules for participation according to consensus mechanisms. In a Blockchain-based supply chain, ledgers enable the involved members to detect any deteriorated data. Thus, Blockchain resolves the issues behind 
the centralized and independent systems, data corruption, hacking, and increases information validity [43]. Moreover, such distributed system can be implemented for traders inexpensively [44].

- Trust: It is the main feature of the DLT that provides subscribers a complete vision of their information. The privacy and anonymity of their accounts are also enabled because of the cryptography system [45]. Each involved subscriber in the DLT system has the exact data and cannot be changed or removed. Thus, the participants' trustworthiness is inevitable within a decentralized supply chain referred to the underlying technology of Blockchain that guarantees the data records' integrity, although some subscribers are dishonest. For this reason, resolving trust and transparency issues is proposed as the primary feature of Blockchain [46], which represents the durability of any supply chain.

- Automation: By relying on predefined rules and conditions, Blockchain applications validate the execution of transactions among two or more intended parties based on smart contracts. The smart contract is a program or script that resides on the main Blockchain Ledger and is executed automatically [47]. It is triggered once some predefined conditions are met. It turns either from a member node or being triggered by another smart contract. Like any other Tx, the smart contract will be published to all the network nodes once deployed. After that, the ledger will be updated if the predefined conditions are met. The supply chain traditional contract is a matter of concern reduced through this automated process where no human intervention is required and no need for trusted intermediaries [48].

\section{3-2-2- Challenges}

Blockchain has another kind of shortcomings as it does not cover all the supply chain requirements. The Blockchainbased supply chain systems encounter new significant issues due to the decentralized nature and the structure of each Blockchain. The scalability is the primary concern of Blockchain applications. It is referred to the delay in responding to the numerous requirements caused by the decentralized P2P system structure. The instability of the network and the low throughput multiply the scalability issue.

Blockchain Tx relies on different factors. Reaching consensus among non-trusted nodes is the core idea of decentralization. The consensus protocol is the essential component of Blockchain that generates and links the blocks to each other and validates Txs within a pure P2P environment without relying on any trusted third party. Despite its precise and vital role in protecting the businesses among non-trusted nodes, the consensus protocol is the major Blockchain component that directly impacts scalability. Furthermore, Blockchain decelerates the application by its design and restrains the Tx with internal cryptographic rules that reduce the Tx propagation speed to an unaccepted rate. Proof of work "POW" [41] is the first consensus protocol of Blockchain limited to seven Txs per second. It is followed by the Proof of Stake "POS" [49] with double speed. Compared with any centralized system, these speeds are frustrated and hinder the progress of the billions of IoT peripherals. Currently, some consensus protocols aim at improving the Tx speed, such as Practical Byzantine Fault Tolerance "PBFT" [50], Proof of Authority" POA" [51], and POS trust "POST" [52] algorithms. The second factor of scalability is the single shared ledger -the Blockchain engine- that contains the blocks, where each block is a set of validated Txs [41]. The processes of block creation and Tx validation consume time and limit/control the propagation of the overall operations to get in the ledger. These processes hinder system performance. The ledger size, the numbers of validator nodes, and the network status of nodes all over the supply chain also directly impact the processing time and performance.

With decentralization, participants are involved in rigorous computing tasks to maintain the distributed ledger. When it comes to IoT devices, it will be a significant challenge in terms of power resources and CPU capacity. Such devices struggle to save energy by sleep mode [53] in idle time, for example. In this manner, they cannot contribute to computing $\mathrm{P} 2 \mathrm{P}$ missions the same way as a dedicated server-miner. Consensus algorithms, such as POW and the cryptographic activities (encrypt, decrypt, hash, etc.) are not applied on most IoT devices [54] that are running on economic power [55], especially in a supply chain that contains millions of resource-limited peripheral devices. Thus, the Tx cost is relatively increased. IoT devices are installed for particular tasks and distributed at a large scale to gather data accurately. The ledger size, which is being stored on every node of the network, increases with time. Thereby, they are very limited in storage capacity $[56,57]$. Most devices are sensors and detectors; thus, it is rare to find an IoT node with accepted memory storage. Hence, reliance on these devices to store the distributed ledger is a considerable challenge that affects the whole system. Furthermore, every Tx is stored permanently on the Blockchain ledger, while storing low-value information is futile. The inability to compact or reduce the ledger's undesired data augments the challenges.

Network infrastructure is likewise a primary factor for a successful Blockchain. All nodes involved in the DLT system should be continuously connected and synchronized to maintain the P2P network. However, high network requirement is inconvenient with IoT's nature. Mainly, most of the supply chains distribute their IoT devices in different geolocations. Providing good throughput is costly and unstable, which poses security risks and/or data instability. Conjointly, Tx fees are still a significant drawback in Blockchain resulting in Tx delays in the process [58]. 


\section{3-3- Typical Supply Chain Requirements}

The three leading technologies that compose the modern supply chain are DLT (mainly Blockchain), supply chain applications, and IoT systems. DLT, IoT, and supply chain platforms altogether compose the robust body of the future supply chain. Thus, considering the combination of these factors [19] while investigating supply chain shortcomings is a must. As we have seen in the previous section, traditional systems encounter many issues and require profound alignment to cope with business expansion. We have also seen that Blockchain is not free of limitations and drawbacks, where regular supply chain integrated with IoT and Blockchain contains drawbacks and limitations [19, 54]. In short, it is like a compatibility matter between the regular supply chain and IoT from one side and between the supply chain and Blockchain from the other side. Hereafter we investigate the relations between supply chain, IoT, and DLT that determine the strength of the modern supply chain. We then list the requirements for the typical platform system and the strategy of the coming works, where scalability, traceability, and overall visibility are the core benefits of a proposed system.

We raise the flag of the typical supply chain so that decentralization is the crucial answer [59, 60], taking into account the diversity of the IoT devices to exempt the weak devices from being charged with heavy tasks. This study is to attain the maximum benefit and advantages from the current DLT and IoT technologies without paying high workload taxes from the peripheral device's resources. The Blockchain requirements for any supply chain system are decentralization, traceability, the immutability of the ledger, fault tolerance, and data security. Besides, smart contract technology running on top of the Blockchain platform introduces process automation and improves the integration of the IoT systems [16]. Table 2 groups the best practices of computing, storage, and Tx fees related to the above DLT problems. The table points out the typical values of the three drawbacks of Blockchain under the typical Blockchain conditions. Each entry of the table determines the perfect situation, which may be out of reach in many cases. However, these inputs assist in constructing the next faultless platform and almost free of limitations. Computing is the primary factor in a DLT P2P system to validate Txs, achieve consensus, and build blocks securely. The P2P system should consider the computing resources of devices not to consume more than a predetermined margin of a participant node resources. Fulfilling the computing tasks requires nodes to be online and synchronized continuously, which is not well-conditioned for most IoT devices in terms of power, network, and device health status.

Besides, the supply chain system is already designed to achieve large complex tasks, so the consensus algorithm and its mining process associated with the DLT system should not affect the production progress. Eliminating high computing is possible, but it supposes conditions and major changes such as diving into permissioned DLT or use specific consensus algorithms such as proof of authority "POA". Storage is another major factor in a successful DLT system. As a P2P system, the ledger is stored basically on peripheral devices that surcharge them by the ever-increasing size. The best practice in a supply chain system is to exempt participants from being charged with a high load.

Table 2. Ideal Blockchain requirements for the supply chain.

\begin{tabular}{ccc}
\hline Computing & Blockchain Requirements & Transaction Fees \\
\hline Not rely on IoT devices & Storage & Consider micropayments \\
Avoid continuously & Not rely on IoT device disks & Feasible transaction fees \\
synchronizations & Accessible anywhere & Not limited to some currencies \\
No high power consumption & High throughput & Consider device status \\
No high network consumption & Secure (data encrypted) & \\
Light mining system & & \\
\hline Light \& secure consensus protocol & & \\
\hline
\end{tabular}

The alternative storage solution should provide extendable storage to avoid the disk space problems and to be located outside the participants' devices (on the cloud, for example) so that the IoT devices can access the data securely and with an acceptable throughput. When it comes to Tx costs, it is highly recommended to consider the micropayment Tx fees. In other terms, the recommended fees system should be very low, feasible for different payment types and currencies. Also, consider the peripheral devices that are working offline or using internet service intermittently to provide the ability to issue offline payments and join the ledger after being online.

Minimizing the impacts of computing, storage, and Tx fees is an uneasy mission. It is based on each use case's requirements and capabilities in managing its parameters. In short, the terms mentioned earlier should be managed so that each case's primary goal is achieved with no negative impacts on the platform. Ideally, the IoT technology should attain all the Blockchain advantages without being charged with computing, storage, and network replication tasks. Currently, this milestone is not well- achieved with the current Blockchain systems. Many projects run on the new supply chain DLT-based concept to somehow introduce many tools in their proposals. We will present these tools in the 
next section to be enrolled in the design of future proposals. Then in section 5 , we focus on the interesting projects, the creators of such tools.

\section{4- Existing Tools to Achieve the Typical Supply Chain Platform}

The proposals of the developed DLT-based supply chain contain some tools and solutions. We investigate these projects and retrieve the existing tools that could be used to fulfill the scalability requirements related to the supply chain progress. It is meaningful to filter them out to design and figure out the future supply chain platform. It is worth noting that no one tool alone helps to achieve the ideal supply chain platform. Instead, a combination of many solutions has an advantage in this situation.

The displayed tools are listed to address the most significant Blockchain problems, where each one treats a single problem. Furthermore, each mechanism has its advantages and drawbacks; thereby, the ideal proposed solution afterward should reap only the pros as maximum as possible. Table 3 depicts the tools' impacts on the three scalability metrics: data size, Tx speed, and Tx cost. Below are the most valuable tools:

Table 3. Tools' impacts on Blockchain Scalability.

\begin{tabular}{ccccccc}
\hline \multirow{2}{*}{ Tools } & \multicolumn{2}{c}{ Data Size } & \multicolumn{2}{c}{ Tx Speed } & \multicolumn{2}{c}{ Tx Cost } \\
\cline { 2 - 6 } & $\begin{array}{c}\text { Tx } \\
\text { Rate }\end{array}$ & $\begin{array}{c}\text { Ledger } \\
\text { Size }\end{array}$ & $\begin{array}{c}\text { Consensus } \\
\text { Algorithm }\end{array}$ & $\begin{array}{c}\text { Block } \\
\text { Structure }\end{array}$ & $\begin{array}{c}\text { Tx } \\
\text { Fees }\end{array}$ & Computing \\
\hline Off-Chain & $\checkmark$ & $\checkmark$ & x & x & x & x \\
Sharding & x & $\checkmark$ & x & x & x & x \\
BaaS & $\checkmark$ & $\checkmark$ & x & x & x & $\checkmark$ \\
Consortium/ Private & x & x & $\checkmark$ & x & $\checkmark$ & $\checkmark$ \\
Modify BC Core & x & x & $\checkmark$ & $\checkmark$ & x & x \\
Editable BC & x & $\checkmark$ & x & x & x & x \\
Alter DLT system & x & x & $\checkmark$ & $\checkmark$ & $\checkmark$ & $\checkmark$ \\
Involvement of IoT device & x & $\checkmark$ & x & x & x & $\checkmark$ \\
\hline
\end{tabular}

\section{a. Off-Chain Solution}

Although Blockchain is an immutable, secure, non-trusted, and linked-time solution for any applications, putting the data on its board, performance and storage issues end up. Off-chain is an exit to benefit from the Blockchain features while the data are processed and stored outside the Blockchain ledger [59]. In this case, Blockchain is responsible for monitoring the overall Tx processes by storing hashed Tx values inside its ledger [60]. Besides, smart contracts can be implemented inside the Blockchain for off-chain services. By doing so, the Blockchain will not be overloaded, and at the same time, data are processed outside the block policies. Thereby this solution provides scalability and data reliability for the off-chain applications while maintaining the decentralization concept. Off-chain can be decentralized but not a DLT-based platform, which can be integrated with other DLT platforms, the case of OriginTrail [61].

\section{b. Sharding Blockchain}

With the increase of participants that share a single Blockchain ledger, distributing blocks among the vast nodes becomes unreliable and affects scalability. The sharding technique is a solution that aims to divide blocks between nodes $[62,63]$. In other words, nodes will be grouped into shards (limit number of nodes), and each shard contains a different part of the ledger. The Tx process, including validation and reaching consensus, and storage location, is limited to the intended shard only. Using this technique in a massive Blockchain environment achieves scalability [64] and prevents high latency [65] following block distribution across the whole nodes.

\section{c. Blockchain as a Service (BaaS)}

Cloud services are adopted to facilitate the IoT functions, storage, and data processing [66], and fog computing technology helps distribute IoT services at a massive scale [67]. However, cryptographic algorithms are not enough to secure such a great workload regarding security and transparency. In addition, with the absence of transparency, data could be corrupted, doubled, or altered due to data spread in different geolocations and distribution on billions of devices. Accordingly, running Blockchain on top of cloud services and edge computing peripherals solves the security and privacy issues throughout the immutable, transparent, and reliable ledger. Besides, cloud plays the role of the big shared extendable storage of the Blockchain instead of relying on the users' devices $[68,60]$. The successful implementation of edge computing servers [67] for a supply chain empowers the IoT devices to exchange data quickly and with minimum latency. 


\section{d. Private/Hybrid Blockchain}

A hybrid Blockchain is a particular Blockchain that lies between private and public Blockchains. It is also called the public-private Blockchain. It is mainly used to restrict the information's visibility in the network. The hybrid Blockchain is represented by partially integrating Blockchain in the workload while keeping a considerable part outside the Blockchain access and control. This type of Blockchain integration is welcomed by systems that do not fully support DLT or insist on keeping part of their application(s) under their centralized control. Multi-chain, such as Cosmos [69], lightning networks, and payment channels use hybrid models. Furthermore, private [70] or consortium Blockchains are closed of permissioned systems where peers deal with Txs upon predefined rules, and the Tx validators are limited to preselected authorized users. The permissioned Blockchain is attractive in terms of performance and scalability since the validators are limited in numbers and device types. With permissioned Blockchain, there is no need for complicated algorithm protocols such as POW or POS. Instead, POA or any other light protocol will be useful and secure in this case.

\section{e. Editable Blockchain}

The well-known immutability and irreversibility features of Blockchain are a two-edged sword. Although these features provide the supply chain with many benefits, they bring some drawbacks. Blockchain suffers from the inability to cope with the high incoming information of the vast IoT environment because of the components that compose its structure. The data recorded in a ledger is permanent, which reflects negatively on the ledger size and pushes all nodes to store the negligible data forever. The feasibility of having an editable Blockchain [71] is not available yet, as it still requires more research. Edit or remove a block(s) due to mistakes, typos, attack impacts, temporary records, or negligible data without breaking the chain enhance Blockchain adoption and scalability. Authors of [71, 72] propose a redactable Blockchain using the Chameleon hash function, which helps to reduce the data size, especially for unimportant data like those related to food after being consumed. This bold move toward editable Blockchain is critical and requires strict governance rules to avoid exploitation and fraud facilities.

\section{f. Blockchain core improvement}

The P2P Blockchain system encumbers IoT devices with computing tasks and high storage demand [40]. In the cryptocurrency field, there are several full nodes prepared with high capacities for the purpose of mining and validation, which is not the case with the massive supply chain IoT devices. Thus, it is required to adapt the main characteristics of Blockchain to boost the association of IoT devices with their circumstances. Minimizing the block size, adjusting the consensus algorithms, and reducing block creation time, are the most changing and adjusting Blockchain areas to fit IoT requirements.

\section{g. Non-Blockchain DLT Platform}

The success of Blockchain encourages researches in this domain. A directed acyclic graph (DAG) [73] technology DLT-based named IOTA [74] is found to tackle IoT devices' scalability and Tx fees where Txs are treated simultaneously. IOTA (or Tangle) is similar to Blockchain in terms of decentralization features, but it differs in structure and behavior. It neither has block, chain, nor Tx fees. The Tx is the only unit in the scene where the node is responsible for validating two previous Txs to get into the Tangle. Running a DAG-based application on IoT devices will overcome the Blockchain shortcomings, but we cannot rely on DAG technology alone (at least for the time being) in managing the whole system. Another DLT system is the Autonomous Decentralized P2P Telemetry (ADEPT) [75], provided by Samsung and IBM. ADEPT categorizes the IoT devices into three types: weak to strong, including light peer, standard peer, and exchange peer. A peer list that is shared among devices permits each device to define its level. The use of lightweight network protocols for IoT devices helps in achieving streaming communication. Also, Hashgraph [76] is a new DLT type, asynchronous Byzantine Fault Tolerance (aBZT) consensus algorithm based on a virtual voting algorithm and the gossip protocol to achieve consensus quickly, fairly, efficiently, and securely.

\section{h. Involvement of IoT Device}

IoT device has two main functions: It captures the data and delivers it to its endpoints. Thus, IoT has two main challenges, the storage and the security of these data. Also, the integration with DLT, end device generates Txs of raw sensory data, verifies Txs, and even mines blocks. It is worth working on improving the IoT manufacturers to be suitable with Blockchain requirements. IOTA [74] categorizes the IoT nodes upon their capacities [77, 78], so there are the full and light nodes. However, the light node still needs to validate Txs to satisfy the system requirements. It is also quite important to mention the necessity of the "IoT for Blockchain" concept. In other terms, to improve the IoT end devices as maximum as possible to fit with DLT requirements in terms of CPU, disks, network, and power. In that way, some of the projects listed in the next section have contributions to the IoT hardware improvements. 


\section{5- Existing DLT-Based Supply Chain Solutions}

The supply chain is the essentiality of all businesses worldwide. So, the DLT integration with the productions and their different partners aims to renovate the global supply chain with the help of smart contracts and other Blockchain features, and IoT technology. Many DLT-based projects aim to provide scalability and reduce the time and cost by investigating new platforms based on DLT. Many challenges encounter these projects, including the type of supply chain business, the main goals of the new platform, in addition to the DLT challenges mentioned above. Ucl CBT report [79] mentions around a hundred projects integrated with DLT and IoT and characterized them upon four natures: healthcare, grocery, fashion, and supply chain. Most of these projects are based on the Ethereum public Blockchain and API interfaces but have no detailed technical references or clear publishments. This paper aims to present considerable studies and projects that invest in the DLT for the supply chain and display their technical part, if any, in the The following subsection. After that, we shed light on the five exciting projects in the following subsection and details their technical contributions.

\section{5-1- DLT-based supply chain}

In this part, we resume the maximum number of projects that interact with DLT and IoT technologies mentioned in previous work [40]. Below are the supply chain projects:

- Shipchain [80] is a fully integrated system of the entire supply chain that is specialized in tracking the shipments from the moment of leaving the factory to the final receiver customer's. Shipchain is running on Ethereum public blockchain using mainly smart contract techniques and side-chain. All the Records are stored on the Ethereum database, while side chain data are stored and validated on the organizations' network for cost-saving purposes. Thereby, the data is located either on Ethereum public ledger or in the side chain ledgers where no intermediary is engaged. Moreover, shipchain contains a web platform that enables shippers to connect directly to carriers without passing by the traditional brokerage models.

- Devery [81] is an open-source protocol based on the Blockchain Ethereum network for verification services. This protocol is used to build applications for verification purposes where sellers can allocate unique signatures to their products. The signatures are stored on the Ethereum ledger and used in verifying a product throughout the application queries. Devery protocol consists of three data structures that interact with Ethereum through DeveryRegistery.sol and DeveryTrust.sol smart contracts. The three data structures are StructApp, unique identifier, and account. StructBrand is used to register a brand public key alongside the unique identifier of an application and store the brand information. Struct Product contains the app account, brand account, and product information. The hash of the product information determines the individual identifier for each product stored on the Blockchain and allows lookup via the check (address item) method. Devery uses the EVE token (Entry Verification Engine) for payments and charges. The consumers of the application service must pay the application host for the product verification service using' Bokky's Token Teleportation Service' (BTTS), which permits consumers to not interact directly with EVE or gas tokens.

- Cargox [82] is a decentralized solution that tackles the bill of lading documents implemented on the Ethereum Blockchain. Cargox is specialized in supply chain logistic trading worldwide. Users communicate via the API interface of the Cargox DApp and create their smart bill of lading. It has payment flexibility, so users can either consume cargo token "CXO" directly or utilize the USD/CXO conversion mechanism.

- CargoCoin [83] is a decentralized supply chain platform based on the Ethereum network that aims to encapsulate all cargoes, transport them into a unique platform, then link them to the intended traders. To achieve this objective, both the services' platform and the smart contracts are utilized within the Blockchain. The platform allows for a range of communication channels between stakeholders involved in the supply chain progression, providing a method of sending/receiving, approving, rejecting or signing documentation.

- Bext360 [84] platform uses the blockchain system to track agriculture products throughout each step in the supply chain. Bext360 provides product traceability and management of payments and smart contracts. It is based on a RESTful API that allows wholesalers and retailers to insert the technology into their own websites, point-of-sale systems, or supply chain management tools.

- Tael (WaBI) [85] is a decentralized application that creates a secure link between partners. It is an independent blockchain installed on user mobile to validate their product through a mobile application. WaBI includes a mining process that incentivizes the users. The incentive process is done throughout the scanning process, where users perform proof of purchase for each scan. The name of Wabi refers to the Walimai organization and supports the "Walimai label," which is applied at a designated 'point of origin' along with the supply chain system. The registered products of the "Walimai system" consume WaBI tokens for their protection.

- TE-FOOD [86] is represented by one ecosystem that involves all partners of the food fabrication (Farmer, producer, 
transporter, and consumer) correspondingly for successful farm-to-table food traceability. TE-FOOD fights against Food frauds and mistrustful supply chains. It introduces a utility token called TFD, blockchain protocol, smart contracts, and 1D/2D and RFID tools for identification purposes. Two types of Blockchain are involved in the progress: the public Ethereum blockchain used for the payment process with TFD token and a second private Blockchain used to store the transactional data. Therefore, supply chain organizations have two types of wallets: Ethereum network wallet, which can be used directly or through the TE-FOOD mobile application, and Transaction wallet on the private network, which the TE-FOOD mobile application can use.

- FarmaTrust [87] provides a solid cloud-based platform that aims to track pharmaceutical products via a supply chain that relies on digital systems to the physical pharmaceuticals. FarmaTrust is based on the Ethereum public Blockchain with a POA consensus algorithm to enhance the scalability. The API and blockchain layers are separated, and the database layer is based on MongoDB and Cassandra.

- BlockGrain [88] is a decentralized platform using Ethereum Blockchain for the agriculture supply chain. BlockGrain is structured into three main layers: public Blockchain, Private Blockchain, and applications. The main data, smart contracts, and Tx Agri tokens are located on the public Blockchain, while buyers utilize the private Blockchain to reduce the costs of both Txs and waiting times associated with a public blockchain. The Blockchains are managed through the applications Layer of the BlockGrain Platform.

- ZERO defects [89] is a DAG-based platform in order to track supply chain products. It is announced through a collaboration between Pickert (ISO certified company) and the IOTA Foundation. Each product is identified using its serial number, and all the data is safe, immutably stored, and accessible in the IOTA Tangle.

- Blockverify [90] is an anti-counterfeit Blockchain-based solution for luxury supply chain items. Blockverify constitutes the combination of Bitcoin and a permissioned Blockchain to store public and private information within the public and private ledgers successively. Each product tracked by Blockverify has a unique special tag along the supply chain, where the customer itself determines the transparency level.

- Chronicled [91] integrates smart tags and the Chronicledapplication to track the physical products and link them to the Blockchain using "identity inlays and tamper-evident cryptographic seals." The Smart Tag is a cryptographically secured chip containing details about the physical good linked with a private key.

- Everledger $[92,93]$ is specialized in protecting the integrity of diamond products using two Blockchain platforms. It uses the private Blockchain 'Hyperledger' and Ethereum public Blockchain to ensure the Tx history's immutability rather than scale up the system.

\section{5-2- Optimized Supply Chain}

The second part of this section elaborates a more detailed inspection of the five DLT- based supply chain projects that optimize parts of the supply chain components. The five DLT-based supply chain projects are involved in the evolution of the modern supply chain integrated with IoT technology. Each project includes a short description and a figure inspired by its whitepaper. Moreover, this study highlights the tools used in each project, the problems encountered, and how far they are practically distinctive from the typical supply chain. Table 4 resumes the essential tools of these projects and their different techniques.

Table 4. Existing tools for the DLT-based supply chain.

\begin{tabular}{|c|c|c|c|c|c|}
\hline Projects & Sharding & Off-Chain & Cloud-Based & $\begin{array}{l}\text { Blockchain Core } \\
\text { Improvement }\end{array}$ & IoT Involvement \\
\hline $\begin{array}{c}\text { Waltonchain } \\
{[52]}\end{array}$ & Not applied & $\begin{array}{l}\text { Subchains are working } \\
\text { as off chain of the } \\
\text { main parentchain }\end{array}$ & Not applied & $\begin{array}{c}\text { Consensus POST } \\
(\mathrm{POS}+\mathrm{POW}+\mathrm{POL})\end{array}$ & RFID enhancements \\
\hline $\begin{array}{l}\text { Origintrail } \\
{[61]}\end{array}$ & Not applied & $\begin{array}{c}\text { ODN:Decentralized } \\
\text { system composed of } 4 \\
\text { node types }\end{array}$ & $\begin{array}{l}\text { Blockchain layer can be } \\
\text { the cloud service }\end{array}$ & Not applied & Not applied \\
\hline $\begin{array}{l}\text { Vechain } \\
\text { [94] }\end{array}$ & $\begin{array}{l}\text { Reed- } \\
\text { Solomon(RS) } \\
\text { algorithm }\end{array}$ & No applied & $\begin{array}{l}\text { Vechainthor is BaaS } \\
\text { for the supply chain } \\
\text { projects }\end{array}$ & $\begin{array}{l}\text { New fields to Tx for-mat to } \\
\text { mitigate a bun- dle of issues Added } \\
\text { new payment method "multi tx } \\
\text { payment" }\end{array}$ & $\begin{array}{l}\text { Upgrade traditional IoT } \\
\text { equipment on the chip level }\end{array}$ \\
\hline $\begin{array}{l}\text { Ambrosus } \\
{[95]}\end{array}$ & Not applied & IPFS(storage) & Not applied & $\begin{array}{l}\text { Custom Ethereum Blockchain } \\
\text { Introduce smart contract } \\
\text { requirement /measurement }\end{array}$ & $\begin{array}{c}\text { Introduce new effective } \\
\text { sensors to trace internal and } \\
\text { external data }\end{array}$ \\
\hline $\begin{array}{c}\text { Modum } \\
\text { [96] }\end{array}$ & Not applied & Not applied & Not applied & Not applied & $\begin{array}{c}\text { Introduce"modum temperature } \\
\text { logger" shipment }\end{array}$ \\
\hline
\end{tabular}




\section{5-2-1- WaltonChain}

WaltonChain [52] is a particular blockchain designed for the supply chain to track the RFID-based Txs by multi partners. As illustrated in Figure 2, it is composed of parent chain and sub-chains with cryptocurrency named WTC running and mined on the parent chain. A sub-chain is working separately after being created and registered inside the parent chain. The parent ledger contains only detailed information related to the sub-chains. Each sub-chain has its ledger and can be created and registered any time under the parent chain network. Parent chain consensus is a combination of POW, POS, and POL. Besides, its block creation time is 60 seconds. Its ledger size is not affected by the number of sub-chains since the parent chain runs independently of sub-chains. Thus, the parent chain is considered scalable and secure. In addition, the smart contract is the foundation of waltonchain that builds and maintains the underlying logic platform.

The waltonchain block contains up to 255 Tx records. It is made up of Block' depth and timestamp, block identity, block account ID and public key, the identity of the previous block and the hash value, the total number of tokens of the Txs contained in the block and byte fee, the Tx information contained in the block, Block payload length and payload hash value, The generated signature of the block, Accumulated coinage difficulty of the block. An updated version of POS called proof of stake \& trust (POST) is used to achieve the consensus of the waltonchain parent blockchain. With the assistance of RFID, Blockchain involved the reputation of nodes to track their behavior and select the honest nodes as coinage nodes. The POST mechanism strengthens the security of the Blockchain. The subchains are free to choose either POS or POST or any other consensus algorithm that fits their application requirements. To create a subchain, the creator consumes WTC on the parent chain, so basic information regarding the new subchain is registered in the current parent block. The creation of a new subchain can be customized based on its nature and conditions. Subchain features mainly include WTC token, subchain token, cross subchain token Txs, smart contract, account control, data storage, etc.

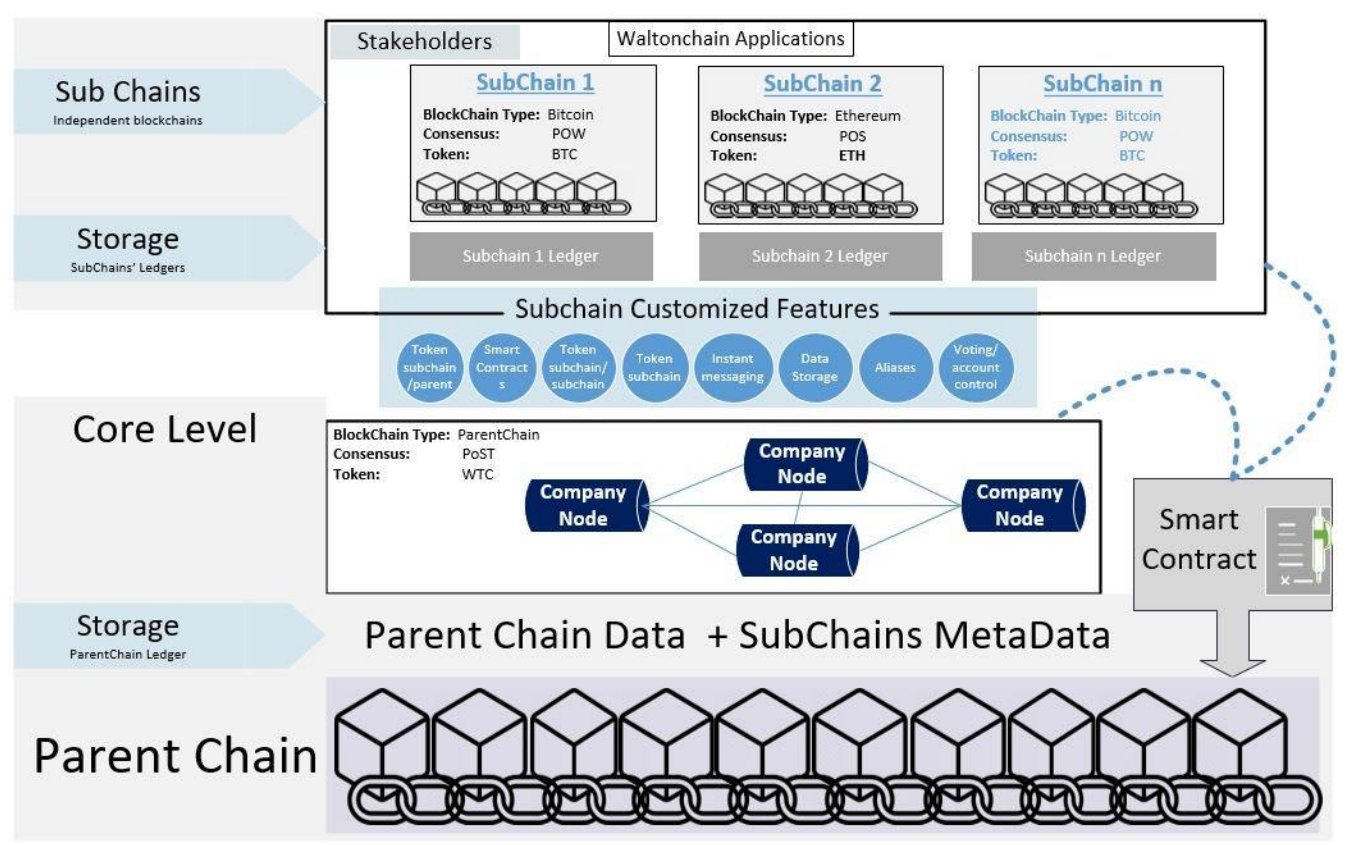

Figure 2. WaltonChain Architecture.

Waltonchain considers the IoT side tool by developing the RFID chips to integrate Blockchain better while tagging assets. But this development is limited to IoT functionality, and it does not tackle the IoT weaknesses mentioned above (computing resources and storage). The block creation time is about one minute, which will inhibit the scalability of the vast IoT involved within the system. The POST consensus algorithm is the waltonchain invented tool to be used within their parentchain platform. The interaction between stakeholders is hard and complicated because subchains have a wide variety of consensus to select. This design does not take into consideration scalability and real-time performance as well as the storage ledger size, network, and computing resources concerns.

\section{5-2-2- OriginTrail}

OriginTrail [61] is a supply chain solution that integrates different partner applications, off-chain networks, and Blockchain. It makes use of different nodes, as shown in Figure 3. Some nodes, which are not DLT-based, implement off-chain within the decentralized environment. Some other nodes are involved in the Blockchain platform. The offchain network is known by ODN (OriginTrail Decentralized Network), which is composed of data and network layers. The architecture is thus the stakeholders' applications, the non-blockchain decentralized ODN, and the Blockchain platform. 
OriginTrail uses the "one step down, one step up" traceability principle to define the stakeholders and their inputs. This principle allows any stakeholder from its position to track back and forward the source and the destination of a product. Using a Blockchain platform is to store the data fingerprint, ensuring integrity and transparency of records and providing an immutable supply chain system. OriginTrail protocol uses the consensus check mechanism to validate data provided by different stakeholders. The consensus check mechanism consists of three steps: Step1: approve the stakeholder by the previous one. Step 2: verify the matching stakeholders. Step 3: verify the matching transactional data and timestamps. It uses the zero-knowledge mechanism to prove private information without revealing them. Four node types are involved in the OriginTrail structures distinguished by their roles: Data provider (DP): represented by the stakeholders, organizations, or consumers with data input to be shared with the supply chain. Data Creator (DC): it is the entry node toward the OriginTrail network. DC nodes receive the data from its provider and link it with data holder nodes. The DC controls and maintains the data process until it is executed. In addition, it checks the availability of the data during service time.

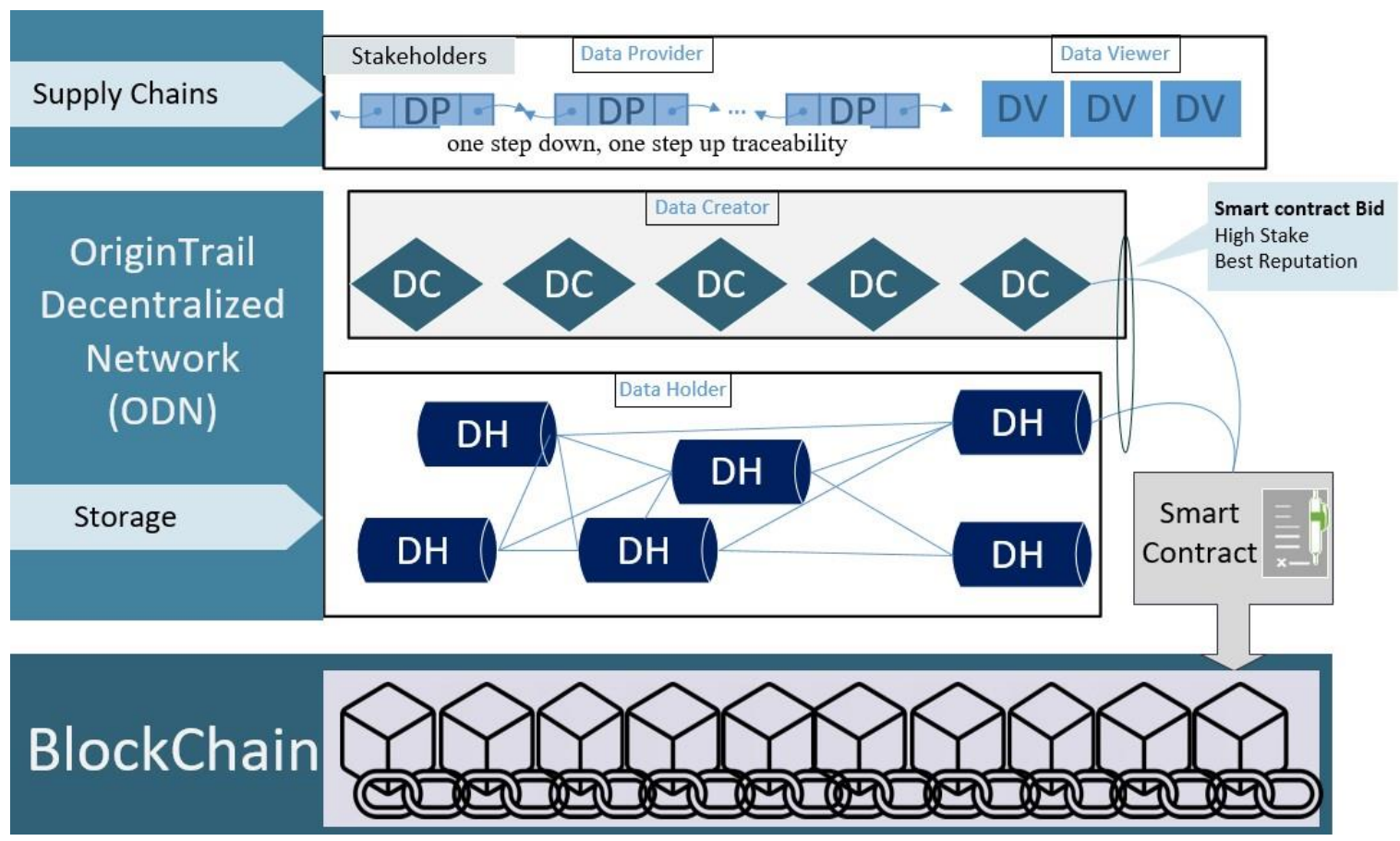

Figure 3. OriginTrail architecture.

Data Holder (DH): DH nodes store the data provided by DC for a specific time and ensure the data will not be altered. DH nodes are highly available to overcome bandwidth bottlenecks and the single point of failure.

DH nodes share the data that is received from DCs in order to reply to some interested parties. DH nodes are compensated by the token" TRAC" for their efforts. A DC is dealing with DH through a smart contract that determines a set of conditions written by DC in addition to the minimum amount of stakes the DH should have. The amount of stakes owned by DHs guarantees its honesty in the execution of the required service. In addition to the stake, the reputation of $\mathrm{DH}$ and other factors are taken into consideration during the selection phase by DC. Data viewer (DV): DV is the entity that requests the data from other nodes with the ability to verify the integrity of the data by running the litigation procedure against the provided data.

The nodes request their services by forming a bid between them (requesters) and the executors (other nodes). Therefore, DCs broadcast their offers to the network that is received by the interested DH nodes. The offer contains the requested services in addition to the criteria of the DH nodes. These criteria are the number of stakes, service price per data unit, the time elapsed to execute the service, and the candidate nodes' reputation. DC selects the storage node (DH) based on its hash value, where the closest hash value is preferable. This selection is automatically made to avoid the DC-specific selection of DH node(s).

Off-chain is the most prominent invented tool of OriginTrail that is represented by the ODN network. The decentralized ODN nodes employ the Blockchain platform to ensure their immutability and data integrity. In this context, Blockchain can be a cloud-based platform that facilitates communication between the different nodes of ODN. The none DLT-based solution provides scalability for the system as it discharges nodes from the computing and power consumption tasks. However, the system is exposed to a vulnerability attack if Blockchain and ODN are disconnected or being forcibly isolated. Besides, the "one step down, one step up" traceability principle provides end-to-end traceability, but a stakeholder is limited to two strides of visibility. 


\section{5-2-3- Vechain}

Vechain [94] is a supply chain solution composed of vechain supply chain projects and vechainthor blockchain-based platform as shown in Figure 4. Vechainthor is an enhanced version of Blockchain. It is forked and improved based on the Ethereum codebase. The following are the enhancements:

1. Tx format includes three new fields: ID, DependsOn, Blockref, and Expiration;

2. Each Tx has its own ID; thereby, the application deals with a single Tx instead of a Tx bundle of Txs;

3. Blockref delivers additional information about the previous, current, and next block. Also, it gives info about Tx creation time. It will be helpful for financial purposes in case of acceptance delay, for example;

4. Expiration is added to the Tx to avoid stacking for a long time. In addition, a Tx can do additional POW to speed up the validation time (it can consume more to track miners' attention, but the extra pow will compensate its loss);

5. Multi-task transaction: a Tx is composed of many small Txs to address the complex business payments. Vechain uses Proof of Authority "POA" as a consensus algorithm that considers both stake and reputation.

The maximum Tx speed announced is 10k Tx per second. As future work, they are looking for involving side chains to enhance the scalability of the vechain system. Vechainthor introduces the new multilayer payment model to ensure the token stability price. Two tokens are utilized to fulfill the Tx process: VET and VTHO. VET is the main token or" smart money" that represents the amounts been held by users. VET generates VTHO to cover the cost of smart contracts and Tx payments. Using this payment method will stabilize the cost of the token. Vechain uses Reed-Solomon (RS) algorithm to shard objects into many parts, and then it reconstructs the data reliably from the remaining drives. Thus Vechain reduces the size of the data significantly. Besides, Vechain considers IoT diversity and categorizes them upon their resources and functionalities in order to best employ their capabilities with Blockchain. Vechain connects the technologies RFID, QR codes, NFC, and bar codes to Blockchain to tag the items by a universally readable identity. This allows tracing the origin of items and prevents counterfeiting since $\mathrm{BC}$ records cannot be alternated or duplicated.

Alter block structure and sharding are the main tools used by Vechain to enhance the Blockchain platform. It also considers the IoT side and categorizes them based on their capabilities. POA consensus algorithm used in Vechain is more diacritical than POW and POS in terms of the validation process, and it does not require computing efforts. However, in a vast supply chain system, the validators become an easy target for adversaries. On the other side, the way the validators are identified renders the platform not entirely decentralized. In an extensive supply chain project where IoT devices spread in many areas, Vechain does not provide a real solution to protect such weak devices from being surcharged by computing tasks.

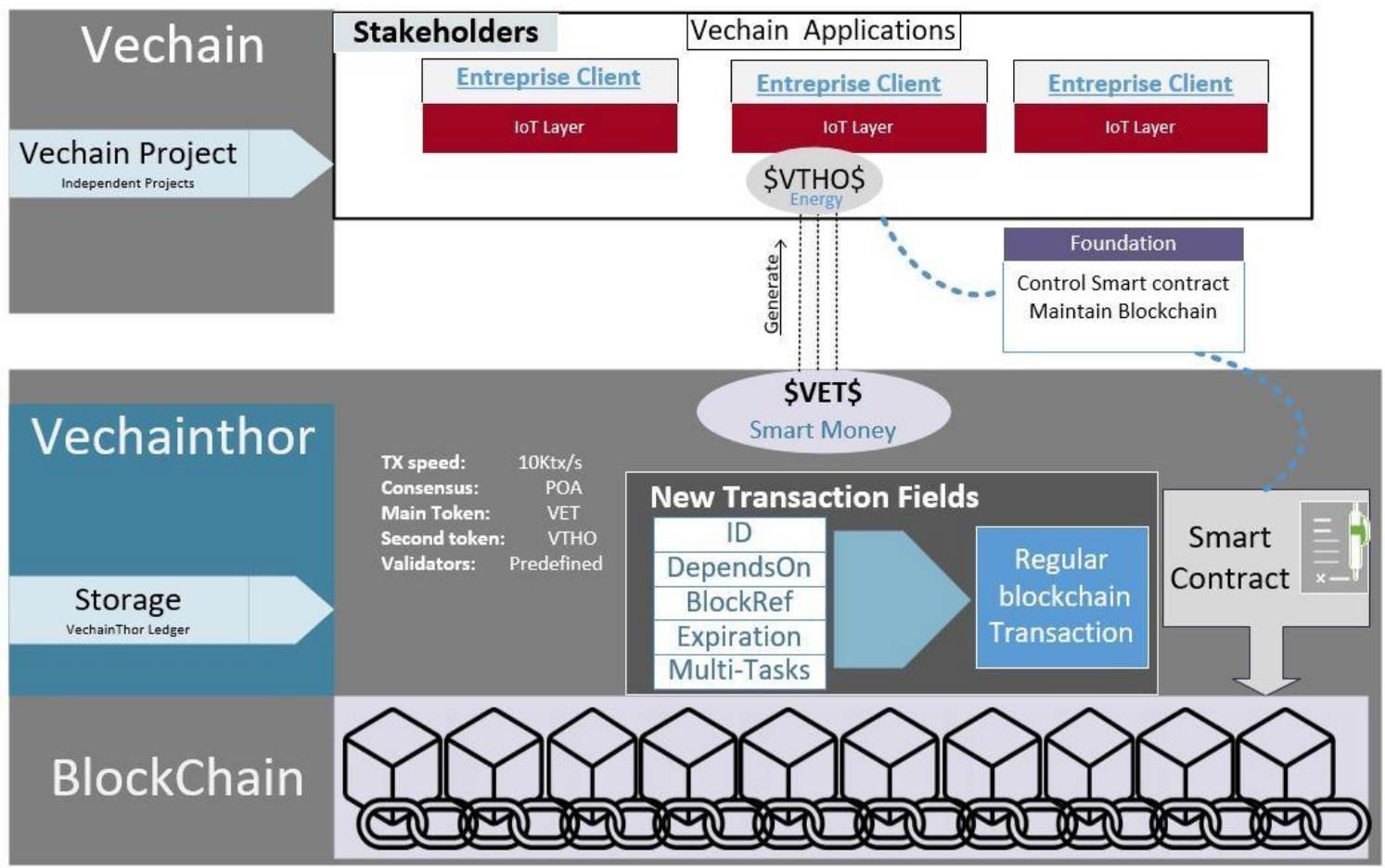

Figure 4. Vechain architecture. 


\section{5-2-4- Ambrosus}

Ambrosus [95] project is to track products throughout their circulation in the market. It is a supply chain Blockchainbased dedicated mainly to protect and control pharmaceutical products and food values. This solution is principally composed of a customized version of Ethereum-Blockchain integrated with a data storage solution named interplanetary file system "IPFS" as illustrated in Figure 5. To avoid the high cost of running the Txs on the main Ethereum platform, Ambrosus develops its independent customized version of Ethereum. Besides, Ambrosus does not rely on Ethereum storage to store the supply chain data as it is limited in capacity. It makes use of IPFS as the main storage for their large Txs to provide scalability and high throughput for the clients. Ambrosus take advantage of the Merkle tree hash cryptography in their transactional processes. With this tree algorithm, users can quickly find their data and filter out the wrong inputs.

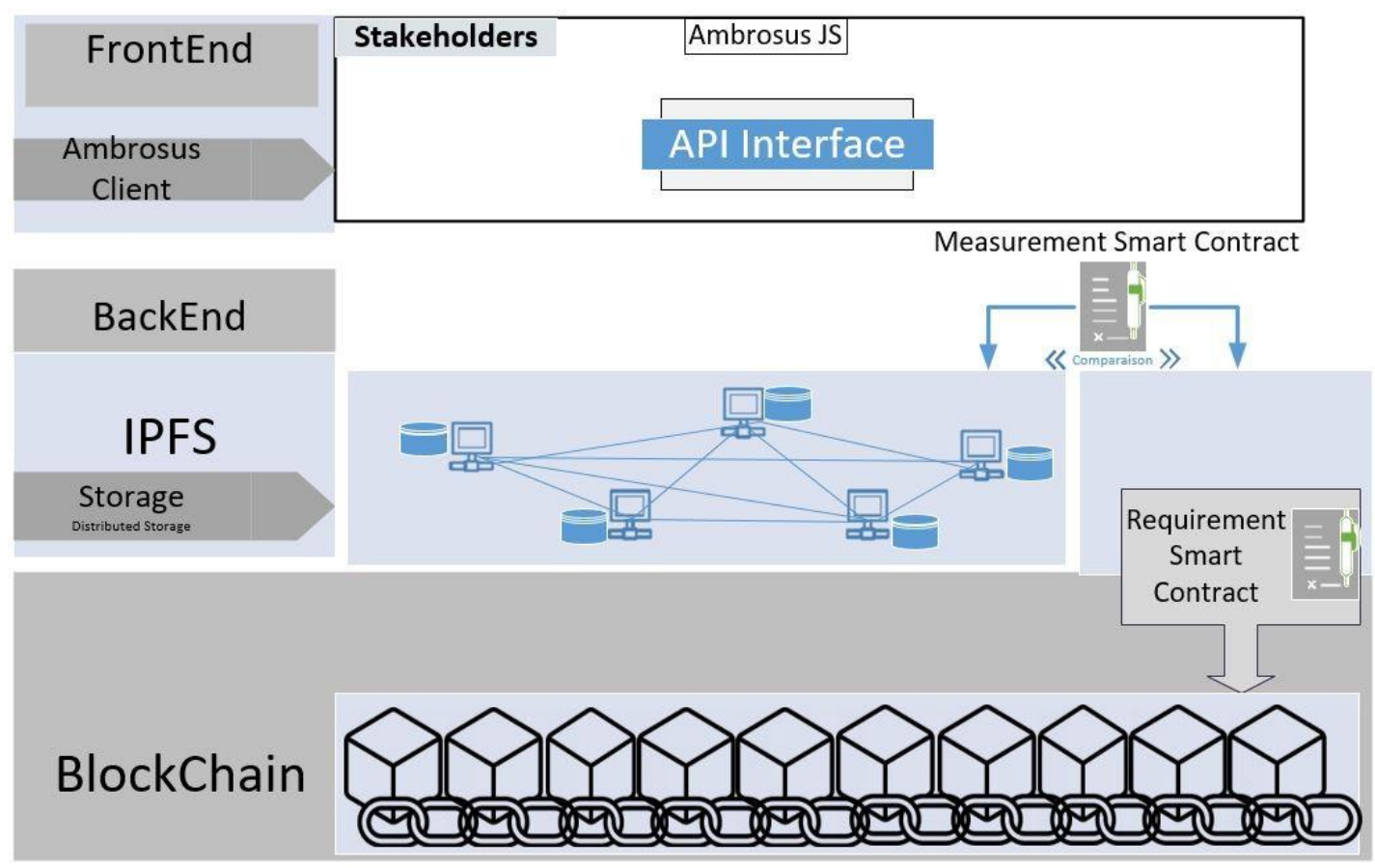

Figure 5. Ambrosus architecture.

Two types of smart contracts are introduced: the requirement smart contract to describe quality standards to be directly compared to items inside the Measurements Smart Contract. The measurement smart contract holds the list of the Merkle tree root hash, ambrosus-certified devices, and the collected attributes throughout the supply chain to note the variation of compositions' quality, if any. The Merkle tree data is uploaded periodically to the main Ethereum network to reduce network problems and improve scalability.

The main structure of the Ambrosus network consists of three main layers: layer 1 contains the Ethereum blockchain and the IPFS storage. Layer 2 represents the supply chains and smart contracts, offline verification, and off-chain operations. Layer 3 represents the application and the Ambrosus javascript protocol. Ambrosus uses IoT hardware and sensors to tag products, thereby tracking goods through the supply chain and ensuring the full integrity of comprehensions.

In addition to the environmental detectors such as temperature, humidity, Ambrosus has an advanced sensitive sensor. Several types of sensors or Biosensors have been developed to detect and analyze particular cases related to food and medicaments. For example, they can detect $\mathrm{pH}$ levels, allergens, DNA, and other types of physical properties.

Ambrosus considers the IoT side by introducing new high-level detector sensors. Besides, it is a customized blockchain that supports off-chain integration. The main ledger stores the Merkle tree roots to ensure that the data cannot be changed once written into a contract. Moreover, the idea behind the Requirements Smart Contract sounds goods, as it determines whether a product continuously meets standards defined by intended participants in the Ambrosus network. This is to control the IoT detectors to get accurate results. On the other side, the structure of the nodes that form the customized Blockchain and the consensus algorithm used or block creation time are questionable. Furthermore, the IPFS storage solution of Ambrosus is not a DLT-based system. It relies on participants' capacities to store the data, including IoT devices. Ambrosus does not respond to computing and storage concerns to enhance IoT integration with the supply chain. 


\section{5-2-5- Modum}

Modum [96] is a supply chain for monitoring solutions that control the distribution of immense volumes of sensitive goods, especially pharmaceutical ones. It comprises the Ethereum blockchain network, the API applications, and a specific sensor called modum temperature logger. Modum architecture constitutes of frontend and backend phases, as illustrated in Figure 6. The backend comprises an Ethereum network, smart contracts, and a specific server connected directly to external users. The frontend comprises sensors and mobile applications connected to the HTTPS server in the backend via REST API and JSON. The SensorTag (Logger) is the top added value that is used to measure the environmental conditions of the shipments. Each logger owns a unique MAC address represented in QR code and each shipment has its unique QR named "track and trace." Both QR codes to be scanned by the user's mobile applications and sent to the server. In case the server is not available, data will be stored on the logger's internal memory. Once received the combination QR codes, the server broadcasts the smart contract, then store the ID of the smart contract on the sensor. Then the client scans the "track and trace" code and requests the temperature measurements from the sensor via BLE "Bluetooth low energy." The smart contract obtains the data for verification purposes and sends back a report to the mobile client. It certifies data authenticity at every change of ownership. The evaluation results are immutably stored in a Blockchain as of proof-of-existence. In this way, open the package to verify the content become useless.

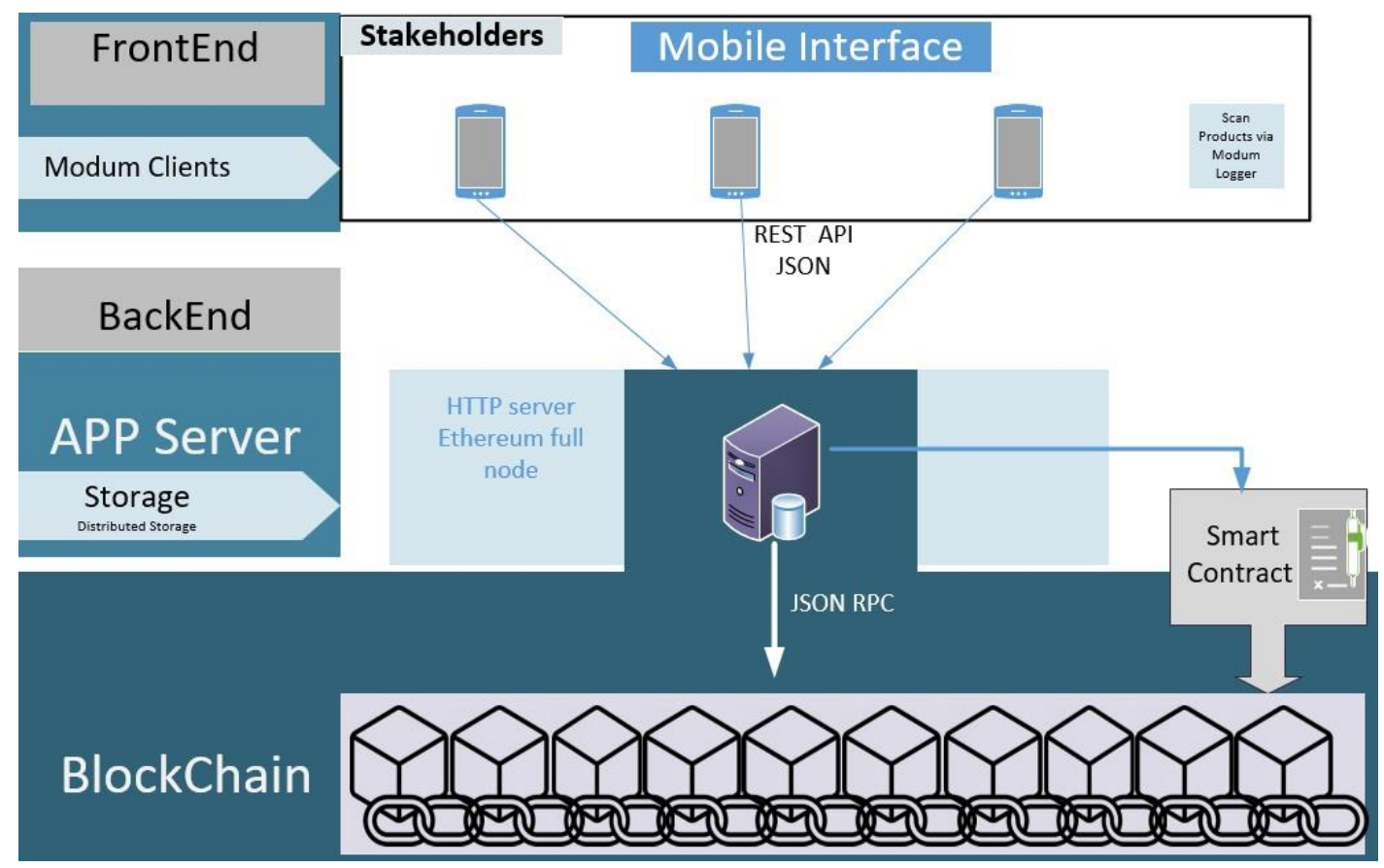

Figure 6. Modum architecture.

The "modum temperature logger" sensor is used to track precisely the temperature of drugs periodically alongside the supply chain. Logger uses the NFC plate to connect with the shipment ID, where each one has a unique smart contract. By using NFC, the Logger alerts the team in case of problems during transport. Furthermore, they can add other sensors related to different monitoring tasks like motion detection for fragile goods, such as evolving their platform to fit different products and partners.

Modum considers the IoT side "tool" by introducing a new sensor involved directly in the tracking process. It uses the public Ethereum for data verification with a specific HTTP server as a full Ethereum node that stores smart contracts and data users. However, this server is considered a single point of failure since it is not decentralized. Furthermore, scalability is not considered and relies on Ethereum efficacity; thereby, improvements over computing and storage are not targeted by this project.

\section{6- Discussion: Supply Chain Scalability}

Previously, we have mentioned various solutions targeting the IoT-based supply chain improvement integrated with Blockchain that enriches the system with trust, complete visibility, and traceability. DLT limitations are manifest to all of these projects, although their technologies and requirements vary markedly. These projects associate the Blockchain within their platforms to overwhelm the downsides and add value to the current DLT systems. The successful integration of DLT with the supply chain is done by employing the available DLT improvements mentioned in this paper as tools (described in section 4) in various ways. 
Table 5. Taxonomy of the supply chain DLT tools.

\begin{tabular}{|c|c|c|}
\hline Layers & Scalability Tools & Supply chain Projects \\
\hline \multirow{10}{*}{ On-chain } & Consensus & Waltonchain [52] \\
\hline & Sharding & Vechain [94](Reed-Solomon algorithm), ProductChain [97] \\
\hline & DAG & ZERO Defects [89], Trade-Markea [98] \\
\hline & Cloud-based & FarmTrust [87] \\
\hline & Transaction format & Vechain [94] \\
\hline & IoT enhancement & WaltonChain [52], Ambrosus [95], Modum [96] \\
\hline & Private/Consortium Blockchain & ProductChain [97] \\
\hline & Side-chain & Waltonchain [52] \\
\hline & Hybrid Blockchain & Modum [96] \\
\hline & Multiple Blockchain Public/Private & $\begin{array}{c}\text { WaltonChain [52], BlockGrain [88], Blockverify [90], } \\
\text { Everledger [93] }\end{array}$ \\
\hline \multirow{5}{*}{ Off-chain } & IPFS & Ambrosus [95] \\
\hline & ODN & OriginTrail [60] \\
\hline & External Storage & ProductChain [97] \\
\hline & PostgreSQL & Modum [96] \\
\hline & BigChainDB & Feng [99] \\
\hline Application & Web interface & Devery [81], Bext360 [84], Modum [96] \\
\hline
\end{tabular}

Scalability is the most crucial requirement of the supply chain metrics that significantly impact the user's Quality of Experience (QoE). It is affected mainly by maximum throughput (transaction speed), data size, and Tx cost metrics illustrated in Table 3 of section 4 . In return, each metric has different root causes behind its failures and different impacts on the overall performance. However, the study sheds light on the positive impacts of the tools on these metrics. Most of these tools are unified under enhancing the scalability behind Blockchain's integration with the supply chain. Each tool is specialized in one of the three scalability metrics mentioned above. Table 5 depicts the approaches' taxonomy to solve the DLT-based supply chain's scalability issue using their tools. Generally, the existing solutions that tackle the scalability issue can be classified into three layers: on-chain, off-chain, and application layers.

\section{6-1- On-Chain Layer}

The on-chain layer solves the drawbacks of the Blockchain system by editing some of its characteristics or introducing multiple Blockchains to distribute the data loads. As the consensus algorithm has the highest impact on scalability, it is considered the primary component to advance the supply chain integration with Blockchain. There are three options to enhance the consensus functionality while keeping the same level of security:

i. Limiting the Blockchain with a few nodes that validate the Txs, which is the case of private Blockchain.

ii. Using consortium Blockchain by changing the consensus algorithm to reduce mining and competitions effects.

iii. Altering the Blockchain entirely by moving to another decentralized platform, such as a DAG-based structure.

Ambrosus, for example, overcomes the scalability issue by reducing the number of validated nodes. It introduces apollo Nodes in the network to validate the Txs only with those nodes with the relevant authority.

In fact, there are many proposed consensus algorithms [100] to enhance the Blockchain mechanism rather than particularizing special servers for validation, which is the case of POA and PBFT (see section 3.2.2). Vechain utilizes the POA consensus algorithm to improve data circulation with a Tx rate of around 10k per second. POA is based on limiting the number of validating nodes in Blockchain. In comparison to PBFT, POA requires fewer message interactions, and therefore it provides high performance. However, both are considered practical in a permissioned Blockchain environment only. Waltonchain introduces another consensus mechanism by combining POW, POS, POL into one consensus algorithm called POST. This algorithm reduces the mining process immensely, saves valuable computing resources, and at the same time, provides a mechanism for selecting the right nodes to strengthen the security of the platform. Also, Waltonchain POST enables communication with any other consensus of their sub-chains which encourages interoperability among supply chain systems. Thus, POST is typical in a supply chain with one central Blockchain where its role is limited to Blockchains' management purpose. It is not designed to deal with huge Tx loads as its block confirmation time is about one minute, hindering IoT devices' proliferation.

The on-chain ledger size is considered the significant challenge of any supply chain where most devices cannot hold such large data volume according to their limited resources. The ledger's data size should be considerably controlled 
from exceeding the limited size. The listed projects earnestly consider controlling the ledger's data size.) Moreover, they (listed projects) propose several solutions to manage this concern. Vechain uses the sharding technique to distribute the ledger volume to many locations using the RS algorithm. Sharding manages the size issues partially because all the data is stored on the Blockchain. In other words, sharding does not solve the volume of the data, but it distributes them. The DAG-based IOTA introduces the snapshot mechanism to remove all records after a particular time. Furthermore, one of the on-chain solutions for scalability and ledger volume is to run a Blockchain in the cloud or use the Blockchain as a service "BaaS."

The participants and the peripheral devices will be out of the computing and data storing tasks as both storage ledger, and computing charges will be offloaded on the cloud. Thus, when Blockchain runs on a Cloud, there is no need to utilize the peripheral device capacity, and there is no need to be fully synchronized. In addition, the edge computing facility over the cloud service enforces scalability, streamlines the data, and empowers IoT devices to exchange their information with low latency. The cloud computing solution behind BaaS facilitates access to a global shared pool of configurable computing resources such as storage services and applications [36]. The stakeholder's cooperation issue is a significant concern in which cloud computing can be considered the important solution [101]. The IoT applications mainly reside in cloud storage and are accessed remotely via mobile application-based running on smartphones or any other devices [102]. Thereby, the cloud improves real-time applications, saves time and cost, and enhances machine-tomachine (M2M) interactions.

Although cloud is considered a suitable place for large ledger size, some data are meaninglessly stored, such as records related to food after consumption. Wherefore, they must be removed. The editing block of a Blockchain ledger breaks the immutability rule of the new decentralization. Nevertheless, Editing blocks is necessary in many cases. Using Chameleon Hash Function [72] could help compress the ledger, but it is still not applied, neither as a pilot nor in production supply chain projects. Suppose the Blockchain becomes editable in the future without risking the integrity of the data. In that case, it will ease off the supply chain from a large amount of undesired/old data, thereby compressing the ledger size as either on-cloud or on local nodes.

\section{6-2-Off-Chain Layer}

The off-chain layer focuses on offloading part of the workload outside the Blockchain platform using either centralized or decentralized systems. In a supply chain, the off-chain tool is proposed within the DLT-based supply chain to reduce Blockchain's storage, save the computing effort, and speed up the Tx process. Moreover, the off-chain is proposed in the supply chain to hide precise information privately and reduce the Tx cost [15]. Technically, Ambrosus uses the IPFS (Interplanetary File System) decentralized storage system to offload the massive data outside the Blockchain so that the cost and the ledger size are reduced. Nevertheless, IPFS is not secure since the IPFS files are accessible to anyone in the network. Also, IPFS is inflexible with corresponding data. Another distributed scenario introduced by OriginTrail called ODN creates an independent decentralized platform to decentralize the Tx processes outside the Blockchain ledger. At the same time, ODN is managed by Blockchain smart contracts to ensure controlling the whole system's integrity. In this regard, all ODN nodes are controlled by Blockchain while offloading a large amount of data outside the DLT ledger. The creation of a decentralized mechanism outside the Blockchain, controlled by the smart contract, is attractive. However, this off-chain platform requires an additional implementation, which is problematic for the additional distributed nodes and their resources. Thus, current off-chain solutions do not help in achieving the typical supply chain. Wherefore, further off-chain enhancements are required to effectively extend its benefits of privacy enablement, high performance, and storage reduction.

\section{6-3- Findings and Open Research}

Based on the above study, many scenarios could be applied to reach a typical supply chain environment by combining different tools listed in Table 3. The eight tools that are introduced above to achieve scalability can be categorized into three types. The first type includes sharding, off-chain tools, IoT involvement, and private/hybrid Blockchain, which solve a specific scalability issue like storage volumes. The second type represents the tools that can commonly be used with different supply chains. It includes BaaS, Editable Blockchain, and Blockchain core improvement. This type can be used with any supply chain as they improve the overall Blockchain platform. The third type is quite different, represented by altering the entire Block system by the DAG-based system. Mainly, the requirements of a supply chain should be settled accurately to select the best-fit tools since no one platform fits with all kinds of businesses. For example, some businesses require real-time data transfer with less computing effort and light ledger size. In contrast, other businesses approve of delayed Txs but require high storage capacity, and so on. 
Table 6. Scalability solutions outside the supply chain.

\begin{tabular}{|c|c|c|c|c|}
\hline Layers & $\begin{array}{l}\text { Scalability } \\
\text { solutions }\end{array}$ & Project & Role and Benefits & Limitations \\
\hline \multirow{14}{*}{ On-Chain } & \multirow{4}{*}{ Block size } & Litecoin [104] & $\begin{array}{l}\text { - Handle large Tx number } \\
\text { - The time block is } 2.5 \text { second } \\
\text { - Throughput is } 56 \text { TPS (Transactions Per Second) }\end{array}$ & - Many orphaned blocks \\
\hline & & $\begin{array}{l}\text { SegWit (Segregated } \\
\text { Witness) [105] }\end{array}$ & $\begin{array}{l}\text { - Remove part of the Transactions } \\
\text { - Divide the block into two: base block and } \\
\text { extended block }\end{array}$ & $\begin{array}{l}\text { - Complexity in the management } \\
\text { of the blocks }\end{array}$ \\
\hline & & Bitcoin Cash [106] & - Increase the block to $32 \mathrm{MB}$ & $\begin{array}{l}\text { - Large block brings back the } \\
\text { centralization }\end{array}$ \\
\hline & & Jidar [107] & $\begin{array}{l}\text { - The node stores the Txs needed only } \\
\text { - keeps Merkle tree image of the whole block }\end{array}$ & $\begin{array}{l}\text {-Not all the data are located on the } \\
\text { node disk }\end{array}$ \\
\hline & \multirow{4}{*}{$\begin{array}{l}\text { Sharding Based } \\
\text { on PoW and } \\
\text { PBFT }\end{array}$} & Elastico [108] & $\begin{array}{l}\text { - Designed for public blockchains } \\
\text { - Tolerate one-fourth fraction of byzantine } \\
\text { faults }\end{array}$ & $\begin{array}{l}\text { - Security challenge } \\
\text { - Throughput } 40 \text { TPS }\end{array}$ \\
\hline & & Omniledger [109] & - Use bias resistant randomness protocol & $\begin{array}{l}\text { - Resilient only } 25 \% \text { to Byzantine } \\
\text { faults }\end{array}$ \\
\hline & & Rapid Chain [65] & $\begin{array}{l}\text { - Kademlia routing algorithm } \\
\text { - Throughput } 7380 \text { TPS }\end{array}$ & $\begin{array}{l}\text { - tolerate up to } 33 \% \text { of } \\
\text { malicious/faulty } \\
\text { - Partitioning attack }\end{array}$ \\
\hline & & Ostraka [110] & $\begin{array}{l}\text { - Shards are the nodes themselves } \\
\text { - Multiple inter/intra shard communication } \\
\text { techniques are used to increase the bandwidth } \\
\text { - Throughput } 400 \mathrm{kTPS}\end{array}$ & -Smart contract limitation \\
\hline & \multirow{3}{*}{$\begin{array}{l}\text { Sharding Based } \\
\text { on PoS and PBFT }\end{array}$} & Zilliqa [111] & $\begin{array}{l}\text { - Execute parallel Txs } \\
\text { - Throughput is } 2828 \text { TPS }\end{array}$ & $\begin{array}{l}\text { - Susceptible to single shard } \\
\text { takeover attacks }\end{array}$ \\
\hline & & Harmony [112] & - Supports state sharding & - Throughput 500 TPS \\
\hline & & Ethereum Sharding 2.0 [113] & $\begin{array}{l}\text { - Executed in three phases: Beacon Chain, } \\
\text { Shard Chain, and State Execution. }\end{array}$ & N/A \\
\hline & \multirow[t]{2}{*}{$\begin{array}{l}\text { Sharding Based } \\
\text { on Consensus }\end{array}$} & Monoxide [114] & $\begin{array}{l}\text { - Linear scaling using asynchronous consensus } \\
\text { zones } \\
\text { - Independent zones, and each one is } \\
\text { responsible for its data }\end{array}$ & $\begin{array}{l}\text { - Asynchronous system hinders the } \\
\text { overall data replication } \\
\text { - Mining and transaction } \\
\text { confirmation are limited to each } \\
\text { zone }\end{array}$ \\
\hline & & Logos [115] & $\begin{array}{l}\text { - Use Axios delegated PBFT consensus } \\
\text { algorithm }\end{array}$ & - Throughput 2500 TPS \\
\hline & DAG & $\begin{array}{l}\text { NXT [116], Nano [117] } \\
\text { Bytball [118], Inclusive } \\
\text { [119], SPECTRE [118], } \\
\text { PHANTOM [120, 121], } \\
\text { Conflux [122],Dagcoin [123] }\end{array}$ & $\begin{array}{l}\text { - Enable low-cost micropayments } \\
\text { - High throughput }\end{array}$ & $\begin{array}{l}\text { - Need high traffic for its } \\
\text { functioning } \\
\text { - Accumulation of unconfirmed } \\
\text { Txs } \\
\text { - Smart contract challenges }\end{array}$ \\
\hline \multirow{3}{*}{ Off-Chain } & \multirow[t]{2}{*}{ Computation } & Truebit [124] & $\begin{array}{l}\text { - Outsource the computations to a verified third- } \\
\text { party } \\
\text { - Third-party called 'solver' is based on smart } \\
\text { contract } \\
\text { - Challenger is another third party to verify the } \\
\text { work done by a solver. }\end{array}$ & \multirow{3}{*}{$\begin{array}{l}\text { - Add complexity to the } \\
\text { implementation }\end{array}$} \\
\hline & & Arbitrum [125] & $\begin{array}{l}\text { - Performs the verification of smart contract off- } \\
\text { chain } \\
\text { - Reducing Ethereum Gas Costs and Increasing } \\
\text { Throughput }\end{array}$ & \\
\hline & Cross-chain & $\begin{array}{l}\text { Polkadot [126] } \\
\text { Cosmos [69] }\end{array}$ & $\begin{array}{l}\text { - Network of many independent blockchains } \\
\text { - Provide interoperability between different } \\
\text { Blockchains } \\
\text { - Achieve high scalability }\end{array}$ & \\
\hline
\end{tabular}

In addition to the improvements/proposals of the above DLT-based projects, several solutions can help achieve scalability if applied to the supply chain. These solutions can be widely used within the public Blockchain, which is the most preferable for the supply chain. In addition, these solutions are categorized into on-chain and off-chain solutions. Table 6, which is inspired from [31], presents the available scalability solutions involved within different approaches. Based on their testing, they have a significant effect on Blockchain performance. The size of the block is one of the major factors since it is related to the number of transactions in a block. The larger the size of the block, the more Txs it receives, leading to high throughput. Another solution is represented by involving sharding technology to mitigate the ledger volume. However, increasing the block size leads to slow propagation and forks in the network. Therefore, different sharding technology $[103,31]$ are surveyed based on the various consensus algorithms.

While many improvements over Blockchain are under development, testing, or creation, it is worth highlight the alternative DLT DAG-based platform shown in Table 6. DAG system such as IOTA is designed to deal with the 
proliferation of IoT devices, and therefore, it is compatible with the modern supply chain. Nevertheless, the DAG-based projects are very few in comparison to the Blockchain-based ones. They are constrained by many drawbacks, like the need for a centralized coordinator (to be removed when the network becomes large enough) and the smart contract challenges. These limitations are not included in Blockchain. From an analysis point of view, amalgamating these two heterogeneous DLTs into one platform helps to attain more significant advantages [60] and advance the IoT integration within the decentralized supply chain. Also, other off-chain implementations of Table 6 are based on computation, and cross-chain can significantly improve scalability and reduce the transaction validation cost.

As a result of this study, there are considerable efforts to address the Blockchain drawbacks. Nevertheless, Blockchain is still not ready to provide high scalability that could fully replace the current centralized and traditional supply chains. Obviously, the aforementioned projects do not provide scalable Blockchain-based solutions referring to the drawbacks they still have not addressed. This paper reveals the current tools that could be integrated differently to positively impact the upcoming new supply chain projects and achieve better performance. Moreover, it is an invitation to deeply study the different solutions/tools mentioned in this paper to be integrated within the future supply chains, considering their drawbacks and limitations.

\section{7- Conclusion}

Blockchain is a promising solution empowered by its trust and collaborative features, satisfying the new supply chain's requirements. Nevertheless, integrating Blockchain with the supply chain brings critical scalability issues, especially in the worldwide systems. This paper highlights the main supply chain challenges and the need for Blockchain to release many existing limitations on the one hand and depicts the Blockchain scalability issues and their effects on the overall DLT-based platforms on the other hand. After that, this paper sets the requirements for the typical DLT platform represented by computing, storage, and Tx cost metrics. In the literature, a considerable number of projects and studies develop Blockchain for the supply chain. This paper technically details the essential recent supply chain projects and brings out their innovative tools, which are employed primarily to mitigate Blockchain scalability. These tools, such as sharding and off-chain solutions, enhance the supply chain performance. However, limitations still exist as the enlisted projects did not satisfy all of the typical DLT requirements. The typical solutions could be inspired by the analyses mentioned above. Combining some existing tools such as integrating DAG with BaaS, merging off-chain and on-chain in a hybrid solution, or editing the core of Blockchain to fit some business criteria, are examples of the suggested solutions. Moreover, other solutions can be applied to the supply chain for further improvements. These solutions include enhancements on the block size level, different sharding mechanisms, multiple DAG platforms, and other off-chain proposals. As a result of this study, the paper calls for further scalability experiments using the existing supply chain innovative tools and the other solutions to reach a typical DLT-based platform suitable for the supply chain.

\section{8- Declarations}

\section{8-1-Author Contributions}

Conceptualization, investigation, methodology, and analysis: H.H., L.S. and A.E.S.; original draft preparation: H.H.; writing - review and editing, H.H., L.S. and A.E.S.; Supervision: L.S., A.E.S. and E.E. All authors have read and agreed to the published version of the manuscript.

\section{8-2-Data Availability Statement}

Data sharing is not applicable to this article.

\section{8-3- Funding}

The authors received no financial support for the research, authorship, and/or publication of this article.

\section{8-4- Conflicts of Interest}

The authors declare that there is no conflict of interests regarding the publication of this manuscript. In addition, the ethical issues, including plagiarism, informed consent, misconduct, data fabrication and/or falsification, double publication and/or submission, and redundancies have been completely observed by the authors.

\section{9- References}

[1] Hugos, Michael "Defining Supply Chain Opportunities." Essentials of Supply Chain Management (March 9, 2012 ): $241-271$. doi:10.1002/9781118386408.ch8.

[2] Barratt, Mark. "Understanding the Meaning of Collaboration in the Supply Chain." Supply Chain Management: An International Journal 9, no. 1 (February 2004): 30-42. doi:10.1108/13598540410517566.

[3] Olorunniwo, Festus O., and Xiaoming Li. "Information Sharing and Collaboration Practices in Reverse Logistics." Supply Chain Management: An International Journal 15, no. 6 (September 28, 2010): 454-462. doi:10.1108/13598541011080437. 
[4] Ramanathan, Usha. "Performance of Supply Chain Collaboration - A Simulation Study." Expert Systems with Applications 41, no. 1 (January 2014): 210-220. doi:10.1016/j.eswa.2013.07.022.

[5] Chen, Ying-Hueih, Tzu-Pei Lin, and David C. Yen. "How to Facilitate Inter-Organizational Knowledge Sharing: The Impact of Trust." Information \& Management 51, no. 5 (July 2014): 568-578. doi:10.1016/j.im.2014.03.007.

[6] Fawcett, Stanley E., Matthew A. Waller, and Amydee M. Fawcett. "Elaborating a Dynamic Systems Theory to Understand Collaborative Inventory Successes and Failures." The International Journal of Logistics Management 21, no. 3 (August 16, 2010): 510-537. doi:10.1108/09574091011089835.

[7] Dey, Nilanjan, Aboul Ella Hassanien, Chintan Bhatt, Amira S. Ashour, and Suresh Chandra Satapathy, eds. "Internet of Things and Big Data Analytics Toward Next-Generation Intelligence." Studies in Big Data (2018). doi:10.1007/978-3-319-60435-0.

[8] Li, YangQun. "An Integrated Platform for the Internet of Things Based on an Open Source Ecosystem." Future Internet 10, no. 11 (October 31, 2018): 105. doi:10.3390/fi10110105.

[9] Griffiths, Francis, and Melanie Ooi. "The Fourth Industrial Revolution - Industry 4.0 and IoT [Trends in Future I\&M]." IEEE Instrumentation \& Measurement Magazine 21, no. 6 (December 2018): 29-43. doi:10.1109/mim.2018.8573590.

[10] Atzori, Luigi, Antonio Iera, and Giacomo Morabito. "The Internet of Things: A Survey." Computer Networks 54 , no. 15 (October 2010): 2787-2805. doi:10.1016/j.comnet.2010.05.010.

[11] Gubbi, Jayavardhana, Rajkumar Buyya, Slaven Marusic, and Marimuthu Palaniswami. "Internet of Things (IoT): A Vision, Architectural Elements, and Future Directions." Future Generation Computer Systems 29, no. 7 (September 2013): 1645-1660. doi:10.1016/j.future.2013.01.010.

[12] Shrouf, F., J. Ordieres, and G. Miragliotta. "Smart Factories in Industry 4.0: A Review of the Concept and of Energy Management Approached in Production Based on the Internet of Things Paradigm." 2014 IEEE International Conference on Industrial Engineering and Engineering Management (December 2014). doi:10.1109/ieem.2014.7058728.

[13] Casino, Fran, Thomas K. Dasaklis, and Constantinos Patsakis. "A Systematic Literature Review of Blockchain-Based Applications: Current Status, Classification and Open Issues." Telematics and Informatics 36 (March 2019): 55-81. doi:10.1016/j.tele.2018.11.006.

[14] Agarwal, Shweta. "Blockchain technology in supply chain and logistics." PhD diss., Massachusetts Institute of Technology, (2018).

[15] Longo, Francesco, Letizia Nicoletti, Antonio Padovano, Gianfranco d' Atri, and Marco Forte. "Blockchain-Enabled Supply Chain: An Experimental Study.” Computers \& Industrial Engineering 136 (October 2019): 57-69. doi:10.1016/j.cie.2019.07.026.

[16] Saberi, Sara, Mahtab Kouhizadeh, Joseph Sarkis, and Lejia Shen. "Blockchain Technology and Its Relationships to Sustainable Supply Chain Management." International Journal of Production Research 57, no. 7 (October 17, 2018): $2117-2135$. doi:10.1080/00207543.2018.1533261.

[17] Wang, Yingli, Jeong Hugh Han, and Paul Beynon-Davies. "Understanding Blockchain Technology for Future Supply Chains: a Systematic Literature Review and Research Agenda." Supply Chain Management: An International Journal 24, no. 1 (January 14, 2019): 62-84. doi:10.1108/scm-03-2018-0148.

[18] Perboli, Guido, Stefano Musso, and Mariangela Rosano. "Blockchain in Logistics and Supply Chain: A Lean Approach for Designing Real-World Use Cases." IEEE Access 6 (2018): 62018-62028. doi:10.1109/access.2018.2875782.

[19] Rejeb, Abderahman, John G. Keogh, and Horst Treiblmaier. "Leveraging the Internet of Things and Blockchain Technology in Supply Chain Management.” Future Internet 11, no. 7 (July 20, 2019): 161. doi:10.3390/fi11070161.

[20] Treiblmaier, Horst, and Roman Beck, eds. "Business Transformation through Blockchain" (2019). doi:10.1007/978-3-31999058-3.

[21] Al-Jaroodi, Jameela, and Nader Mohamed. "Blockchain in Industries: A Survey." IEEE Access 7 (2019): $36500-36515$. doi:10.1109/access.2019.2903554.

[22] Hald, Kim Sundtoft, and Aseem Kinra. "How the Blockchain Enables and Constrains Supply Chain Performance." International Journal of Physical Distribution \& Logistics Management 49, no. 4 (June 13, 2019): 376-397. doi:10.1108/ijpdlm-02-20190063.

[23] Wang, Yingli, Meita Singgih, Jingyao Wang, and Mihaela Rit. "Making Sense of Blockchain Technology: How Will It Transform Supply Chains?" International Journal of Production Economics 211 (May 2019): 221-236. doi:10.1016/j.ijpe.2019.02.002.

[24] Pournader, Mehrdokht, Yangyan Shi, Stefan Seuring, and S.C. Lenny Koh. "Blockchain Applications in Supply Chains, Transport and Logistics: a Systematic Review of the Literature." International Journal of Production Research 58, no. 7 (August 11, 2019): 2063-2081. doi:10.1080/00207543.2019.1650976. 
[25] Azzi, Rita, Rima Kilany Chamoun, and Maria Sokhn. "The Power of a Blockchain-Based Supply Chain." Computers \& Industrial Engineering 135 (September 2019): 582-592. doi:10.1016/j.cie.2019.06.042.

[26] Scully, Padraig, and Michael Hobig. "Exploring the Impact of Blockchain on Digitized Supply Chain Flows: A Literature Review." 2019 Sixth International Conference on Software Defined Systems (SDS) (June 2019). doi:10.1109/sds.2019.8768573.

[27] Tribis, Youness, Abdelali El Bouchti, and Houssine Bouayad. "Supply Chain Management Based on Blockchain: A Systematic Mapping Study." Edited by B. Abou El Majd and H. El Ghazi. MATEC Web of Conferences 200 (2018): 00020. doi:10.1051/matecconf/201820000020.

[28] Helo, Petri, and Yuqiuge Hao. "Blockchains in Operations and Supply Chains: A Model and Reference Implementation." Computers \& Industrial Engineering 136 (October 2019): 242-251. doi:10.1016/j.cie.2019.07.023.

[29] Saberi, Sara, Mahtab Kouhizadeh, and Joseph Sarkis. "Blockchains and the Supply Chain: Findings from a Broad Study of Practitioners." IEEE Engineering Management Review 47, no. 3 (September 1, 2019): 95-103. doi:10.1109/emr.2019.2928264.

[30] Gonczol, Peter, Panagiota Katsikouli, Lasse Herskind, and Nicola Dragoni. "Blockchain Implementations and Use Cases for Supply Chains-A Survey." IEEE Access 8 (2020): 11856-11871. doi:10.1109/access.2020.2964880.

[31] Yadav, Jyoti, and Ranjana Shevkar. "Performance-Based Analysis of Blockchain Scalability Metric." Tehnički Glasnik 15, no. 1 (March 4, 2021): 133-142. doi:10.31803/tg-20210205103310.

[32] Hsu, Cheng. "Service Science: Design for Scaling and Transformation" (February 2009). doi:10.1142/7098.

[33] Lev, Baruch, Suresh Radhakrishnan, and Weining Zhang. "Organization Capital." Abacus 45, no. 3 (September 2009): $275-298$. doi:10.1111/j.1467-6281.2009.00289.x.

[34] He, Longfei, Mei Xue, and Bin Gu. "Internet-of-Things Enabled Supply Chain Planning and Coordination with Big Data Services: Certain Theoretic Implications." Journal of Management Science and Engineering 5, no. 1 (March 2020): 1-22. doi:10.1016/j.jmse.2020.03.002.

[35] Liu, Li, Xinrui Liu, and Xinyu Li. "Cloud-Based Service Composition Architecture for Internet of Things." Communications in Computer and Information Science (2012): 559-564. doi:10.1007/978-3-642-32427-7_80.

[36] Lee, In, and Kyoochun Lee. "The Internet of Things (IoT): Applications, Investments, and Challenges for Enterprises." Business Horizons 58, no. 4 (July 2015): 431-440. doi:10.1016/j.bushor.2015.03.008.

[37] Tzounis, Antonis, Nikolaos Katsoulas, Thomas Bartzanas, and Constantinos Kittas. "Internet of Things in Agriculture, Recent Advances and Future Challenges." Biosystems Engineering 164 (December 2017): 31-48. doi:10.1016/j.biosystemseng.2017.09.007.

[38] Manyika, James, Michael Chui, Peter Bisson, Jonathan Woetzel, Richard Dobbs, Jacques Bughin, and Dan Aharon. "The Internet of Things: Mapping the value beyond the hype." (2015).

[39] Haswell, Holli, and M. Storgaard. "Maersk and IBM unveil first industry-wide cross-border supply chain solution on blockchain." IBM. Available online: http://www-03.ibm.com/press/us/en/pressrelease/51712.wss. (accessed on May 2021).

[40] Hellani, Houssein, Layth Sliman, Abed Ellatif Samhat, and Ernesto Exposito. "On Blockchain Integration with Supply Chain: Overview on Data Transparency.” Logistics 5, no. 3 (July 2, 2021): 46. doi:10.3390/logistics5030046.

[41] Hellani, Hussein, Abed Ellatif Samhat, Maroun Chamoun, Hussein El Ghor, and Ahmed Serhrouchni. "On BlockChain Technology: Overview of Bitcoin and Future Insights." 2018 IEEE International Multidisciplinary Conference on Engineering Technology (IMCET) (November 2018). doi:10.1109/imcet.2018.8603029.

[42] Rauchs, Michel, Andrew Glidden, Brian Gordon, Gina C. Pieters, Martino Recanatini, François Rostand, Kathryn Vagneur, and Bryan Zheng Zhang. "Distributed Ledger Technology Systems: A Conceptual Framework." SSRN Electronic Journal (2018). doi:10.2139/ssrn.3230013.

[43] Feng Tian. "An Agri-Food Supply Chain Traceability System for China Based on RFID \& Blockchain Technology." 2016 13th International Conference on Service Systems and Service Management (ICSSSM) (June 2016). doi:10.1109/icsssm.2016.7538424.

[44] Bocek, Thomas, Bruno B. Rodrigues, Tim Strasser, and Burkhard Stiller. "Blockchains Everywhere - a Use-Case of Blockchains in the Pharma Supply-Chain." 2017 IFIP/IEEE Symposium on Integrated Network and Service Management (IM) (May 2017). doi:10.23919/inm.2017.7987376.

[45] MCrosby, Michael, Pradan Pattanayak, Sanjeev Verma, and Vignesh Kalyanaraman. "Blockchain technology: Beyond bitcoin." Applied Innovation 2, no. 6-10 (2016): 71. Available online: https://j2-capital.com/wp-content/uploads/2017/11/AIR-2016Blockchain.pdf (accessed on May 2021).

[46] Casey, Michael J., and Pindar Wong. "Global supply chains are about to get better, thanks to blockchain." Harvard business review 13 (2017): 1-6. 
[47] Peters, Gareth William, and Efstathios Panayi. "Understanding Modern Banking Ledgers through Blockchain Technologies: Future of Transaction Processing and Smart Contracts on the Internet of Money." SSRN Electronic Journal (2015). doi:10.2139/ssrn.2692487.

[48] Bocek, Thomas, and Burkhard Stiller. "Smart Contracts - Blockchains in the Wings." Digital Marketplaces Unleashed (September 15, 2017): 169-184. doi:10.1007/978-3-662-49275-8_19.

[49] Wood, Gavin. "Ethereum: A secure decentralised generalised transaction ledger." Ethereum project yellow paper 151, no. 2014 (2014): 1-32.

[50] Jiang, Yiming, Chenxu Wang, Yawei Wang, and Lang Gao. "A Cross-Chain Solution to Integrating Multiple Blockchains for IoT Data Management." Sensors 19, no. 9 (May 1, 2019): 2042. doi:10.3390/s19092042.

[51] De Angelis, Stefano, Leonardo Aniello, Roberto Baldoni, Federico Lombardi, Andrea Margheri, and Vladimiro Sassone. "PBFT vs proof-of-authority: Applying the CAP theorem to permissioned blockchain." (2018).

[52] Waltonchain whitepaper, Available online: https://www.waltonchain.org/upload/1507947 652573.pdf. (accessed on 05 May 2021)

[53] Liang, Jia-Ming, Jen-Jee Chen, Hung-Hsin Cheng, and Yu-Chee Tseng. "An Energy-Efficient Sleep Scheduling With QoS Consideration in 3GPP LTE-Advanced Networks for Internet of Things." IEEE Journal on Emerging and Selected Topics in Circuits and Systems 3, no. 1 (March 2013): 13-22. doi:10.1109/jetcas.2013.2243631.

[54] Wang, Xu, Xuan Zha, Wei Ni, Ren Ping Liu, Y. Jay Guo, Xinxin Niu, and Kangfeng Zheng. "Survey on Blockchain for Internet of Things." Computer Communications 136 (February 2019): 10-29. doi:10.1016/j.comcom.2019.01.006.

[55] Westermann, Benedikt, Danilo Gligoroski, and Svein Knapskog. "Comparison of the Power Consumption of the 2nd Round SHA-3 Candidates." ICT Innovations 2010 (2011): 102-113. doi:10.1007/978-3-642-19325-5_11.

[56] Hang, Lei, and Do-Hyeun Kim. "Design and Implementation of an Integrated IoT Blockchain Platform for Sensing Data Integrity." Sensors 19, no. 10 (May 14, 2019): 2228. doi:10.3390/s19102228.

[57] Sun, Xiang, and Nirwan Ansari. "Dynamic Resource Caching in the IoT Application Layer for Smart Cities." IEEE Internet of Things Journal 5, no. 2 (April 2018): 606-613. doi:10.1109/jiot.2017.2764418.

[58] Xu, Xiwei, Ingo Weber, and Mark Staples. "Architecture for Blockchain Applications" (2019). doi:10.1007/978-3-030-03035-3.

[59] Dujak, Davor, and Domagoj Sajter. "Blockchain Applications in Supply Chain.” EcoProduction (June 15, 2018): 21-46. doi:10.1007/978-3-319-91668-2_2.

[60] Hellani, Hussein, Layth Sliman, Motaz Ben Hassine, Abed Ellatif Samhat, Ernesto Exposito, and Mourad Kmimech. "Tangle the Blockchain: Toward IOTA and Blockchain Integration for IoT Environment." Advances in Intelligent Systems and Computing (August 13, 2020): 429-440. doi:10.1007/978-3-030-49336-3_42.

[61] Origintrail whitepaper, Available online: https://origintrail.io/storage/documents/OriginTrail-White-Paper.pdf. (accessed on 05 May 2021).

[62] Luu, Loi, Viswesh Narayanan, Chaodong Zheng, Kunal Baweja, Seth Gilbert, and Prateek Saxena. "A Secure Sharding Protocol For Open Blockchains." Proceedings of the 2016 ACM SIGSAC Conference on Computer and Communications Security (October 24, 2016). doi:10.1145/2976749.2978389.

[63] Gencer, Adem Efe, Robbert van Renesse, and Emin Gün Sirer. "Short Paper: Service-Oriented Sharding for Blockchains." Lecture Notes in Computer Science (2017): 393-401. doi:10.1007/978-3-319-70972-7_22.

[64] Dang, Hung, Tien Tuan Anh Dinh, Dumitrel Loghin, Ee-Chien Chang, Qian Lin, and Beng Chin Ooi. "Towards Scaling Blockchain Systems via Sharding." Proceedings of the 2019 International Conference on Management of Data (June 25, 2019). doi:10.1145/3299869.3319889.

[65] Zamani, Mahdi, Mahnush Movahedi, and Mariana Raykova. "RapidChain." Proceedings of the 2018 ACM SIGSAC Conference on Computer and Communications Security (January 15, 2018). doi:10.1145/3243734.3243853.

[66] Liu, Yu, Beibei Dong, Benzhen Guo, Jingjing Yang, and Wei Peng. "Combination of Cloud Computing and Internet of Things (IOT) in Medical Monitoring Systems." International Journal of Hybrid Information Technology 8, no. 12 (December 31, 2015): 367-376. doi:10.14257/ijhit.2015.8.12.28.

[67] Yang, Ruizhe, F. Richard Yu, Pengbo Si, Zhaoxin Yang, and Yanhua Zhang. "Integrated Blockchain and Edge Computing Systems: A Survey, Some Research Issues and Challenges." IEEE Communications Surveys \& Tutorials 21, no. 2 (2019): 15081532. doi:10.1109/comst.2019.2894727.

[68] Pohrmen, Fabiola Hazel, Rohit Kumar Das, and Goutam Saha. "Blockchain-based Security Aspects in Heterogeneous Internetof-Things Networks: A Survey." Transactions on Emerging Telecommunications Technologies 30, no. 10 (September 6, 2019). doi:10.1002/ett.3741. 
[69] Kwon, Jae, and Ethan Buchman. "A network of distributed ledgers." Cosmos, dated (2018): 1-41. Available online: https://cosmos.net- work/whitepaper. (accessed on 05 May 2021).

[70] Sankar, Lakshmi Siva, M. Sindhu, and M. Sethumadhavan. "Survey of Consensus Protocols on Blockchain Applications." 2017 4th International Conference on Advanced Computing and Communication Systems (ICACCS) (January 2017). doi:10.1109/icaccs.2017.8014672.

[71] Ateniese, Giuseppe, Bernardo Magri, Daniele Venturi, and Ewerton Andrade. "Redactable Blockchain - or - Rewriting History in Bitcoin and Friends." 2017 IEEE European Symposium on Security and Privacy (EuroS\&P) (April 2017). doi:10.1109/eurosp.2017.37.

[72] Ashritha, Kondapally, M. Sindhu, and K.V. Lakshmy. "Redactable Blockchain Using Enhanced Chameleon Hash Function." 2019 5th International Conference on Advanced Computing \& Communication Systems (ICACCS) (March 2019). doi:10.1109/icaccs.2019.8728524.

[73] Hellani, Houssein, Layth Sliman, Abed Ellatif Samhat, and Ernesto Exposito. "Computing Resource Allocation Scheme for DAG-Based IOTA Nodes.” Sensors 21, no. 14 (July 9, 2021): 4703. doi:10.3390/s21144703.

[74] Popov, Serguei, Hans Moog, Darcy Camargo, Angelo Capossele, Vassil Dimitrov, Alon Gal, Andrew Greve et al. "The coordicide." Accessed Jan (2020): 1-30.

[75] Panikkar, Sanjay, Sumabala Nair, Paul Brody, and Veena Pureswaran. "Adept: An iot practitioner perspective." Draft Copy for Advance Review, IBM (2015).

[76] Baird, Leemon, Mance Harmon, and Paul Madsen. "Hedera: A governing council \& public hashgraph network." The trust layer of the internet, whitepaper 1 (2018): 1-97.

[77] J. McKinney, Light client protocol, Available online: https://github.com/ethereum/wiki/wiki/Light-client-protocol (accessed on 05 May 2021).

[78] Iota light nodes vs full nodes, Available online: https://iota.readme.io/v1.2.0/docs/light-vsfull-node (accessed on 05 May 2021).

[79] Ucl CBT report, Available online: https://bit.ly/3dO6KeF (accessed on 05 May 2021).

[80] Shipchain whitepaper (2017), Available online: https://shipchain.io (accessed on 05 May 2021).

[81] Devery whitepaper (2017-2020), Available online: https://devery.io/ (accessed on 05 May 2021).

[82] Cargox whitepaper (2015), Available online: https://cargox.io/ (accessed on 05 May 2021).

[83] Cargocoin whitepaper Available online: https://thecargocoin.com/ (accessed on 05 May 2021).

[84] Bext360, Available online: https://www.bext360.com/ (accessed on 05 May 2021).

[85] Tael wabi, (2017), Available online: https://www.taelpay.com/ (accessed on 05 May 2021).

[86] Te-food whitepaper, Available online: https://www.te-food.com/te-food-white-paper.pdf. (accessed on 05 May 2021).

[87] Farmtrust whitepaper (2019), Available online: https://www.farmatrust.com/ (accessed on 05 May 2021).

[88] Blockgrain whitepaper (2018), Available online: https://pages.agrichain.com. (accessed on 05 May 2021).

[89] Iota blog (2020), Available online: https://blog.iota.org/zero-defects-digital-twins-and-iota-8568f54c3925. (accessed on 05 May 2021).

[90] Bocek, Thomas, Bruno B. Rodrigues, Tim Strasser, and Burkhard Stiller. "Blockchains Everywhere - a Use-Case of Blockchains in the Pharma Supply-Chain.” 2017 IFIP/IEEE Symposium on Integrated Network and Service Management (IM) (May 2017). doi:10.23919/inm.2017.7987376.

[91] Chronicled inc., Business to business service in San Francisco, California linking the physical world to the blockchain, 2016, Available online: http://www.chronicled. com/. (accessed on 05 May 2021)

[92] Lomas, Natasha. "Everledger is using blockchain to combat fraud, starting with diamonds." Tech Crunch 29 (2015).

[93] G.D.C. Exchange, White paper transforming diamonds into a new financial asset class (2018).

[94] Vechain whitepaper, Available online: https://vechain.com. (accessed on 05 May 2021).

[95] Ambrosus whitepaper, Available online: https://whitepaperdatabase.com/ambrosus-amb-whitepaper. (accessed on 05 May 2021).

[96] Modum whitepaper, Available online: https://whitepaper.io/document/213/modum-whitepaper. (accessed on 05 May 2021)

[97] Malik, Sidra, Salil S. Kanhere, and Raja Jurdak. "ProductChain: Scalable Blockchain Framework to Support Provenance in Supply Chains." 2018 IEEE 17th International Symposium on Network Computing and Applications (NCA) (November 2018). doi:10.1109/nca.2018.8548322. 
[98] Trademarkea, Available online: www.trademarkea.com. (accessed on 05 May 2021)

[99] Feng Tian. "An Agri-Food Supply Chain Traceability System for China Based on RFID \& Blockchain Technology." 2016 13th International Conference on Service Systems and Service Management (ICSSSM) (June 2016). doi:10.1109/icsssm.2016.7538424.

[100] Kim, Soohyeong, Yongseok Kwon, and Sunghyun Cho. "A Survey of Scalability Solutions on Blockchain." 2018 International Conference on Information and Communication Technology Convergence (ICTC) (October 2018). doi:10.1109/ictc.2018.8539529.

[101] Li, Xuefeng, Ying Wang, and Xi Chen. "Cold Chain Logistics System Based on Cloud Computing." Concurrency and Computation: Practice and Experience 24, no. 17 (September 22, 2011): 2138-2150. doi:10.1002/cpe.1840.

[102] Saragih, L. Ranjaliba, M. Dachyar, Teuku Yuri M. Zagloel, and Muhammad Satar. "The Industrial IoT for Nusantara.” 2018 IEEE International Conference on Internet of Things and Intelligence System (IOTAIS) (November 2018). doi:10.1109/iotais.2018.8600860.

[103] Yu, Guangsheng, Xu Wang, Kan Yu, Wei Ni, J. Andrew Zhang, and Ren Ping Liu. "Survey: Sharding in Blockchains." IEEE Access 8 (2020): 14155-14181. doi:10.1109/access.2020.2965147.

[104] Litecoin: The Cryptocurrency for Payments, Available online: https://litecoin.org/ (accessed on 05 May 2021)

[105] Lombrozo, Eric, Johnson Lau, and Pieter Wuille. "Segregated witness (consensus layer)." Bitcoin Core Develop. Team, Tech. Rep. BIP 141 (2015).

[106] "Bitcoin cash" Available online: https://news.bitcoin.com/fork-watch-first-bitcoin-cash-block-mined. (accessed on 05 May 2021)

[107] Dai, Xiaohai, Jiang Xiao, Wenhui Yang, Chaofan Wang, and Hai Jin. "Jidar: A Jigsaw-Like Data Reduction Approach without Trust Assumptions for Bitcoin System.”2019 IEEE 39th International Conference on Distributed Computing Systems (ICDCS) (July 2019). doi:10.1109/icdcs.2019.00132.

[108] Luu, Loi, Viswesh Narayanan, Chaodong Zheng, Kunal Baweja, Seth Gilbert, and Prateek Saxena. "A Secure Sharding Protocol for Open Blockchains." Proceedings of the 2016 ACM SIGSAC Conference on Computer and Communications Security (October 24, 2016). doi:10.1145/2976749.2978389.

[109] Kokoris-Kogias, Eleftherios, Philipp Jovanovic, Linus Gasser, Nicolas Gailly, Ewa Syta, and Bryan Ford. "OmniLedger: A Secure, Scale-Out, Decentralized Ledger via Sharding.” 2018 IEEE Symposium on Security and Privacy (SP) (May 2018). doi:10.1109/sp.2018.000-5.

[110] Manuskin, Alex, Michael Mirkin, and Ittay Eyal. “Ostraka: Secure Blockchain Scaling by Node Sharding.” 2020 IEEE European Symposium on Security and Privacy Workshops (EuroS\&PW) (September 2020). doi:10.1109/eurospw51379.2020.00060.

[111] Team, Hercules Core. “Hercules Whitepaper.” SSRN Electronic Journal (2018). doi:10.2139/ssrn.3442330.

[112] "Harmony" Available online: https://harmony.one (accessed on 05 May 2021)

[113] "Ethereum Sharding 2.0" Buterin. Ethereum Sharding FAQ. Available online: https://github.com/ethereum/wiki/wiki/ Sharding-FAQ. (accessed on 05 May 2021)

[114] Wang, Jiaping, and Hao Wang. "Monoxide: Scale out blockchains with asynchronous consensus zones." In 16th (USENIX) Symposium on Networked Systems Design and Implementation, NSDI, (2019): 95-112.

[115] "Logos" Available online: https://cryptodiffer.com/logos-network-ico (accessed on 05 April 2021).

[116] "NXT" whitepaper Available online: https://whitepaper.io/coin/nxt (accessed on 05 May 2021).

[117] LeMahieu, Colin. "Nano: A feeless distributed cryptocurrency network." Nano 16 (2018): 17. Available online: https://nano.org/en/whitepaper (accessed on 05 April 2021).

[118] Churyumov, Anton. "Byteball: A decentralized system for storage and transfer of value." (2016):1-49. Available online: https://byteball.org/ Byteball.pdf (accessed on 05 May 2021).

[119] Lewenberg, Yoad, Yonatan Sompolinsky, and Aviv Zohar. "Inclusive Block Chain Protocols." Lecture Notes in Computer Science (2015): 528-547. doi:10.1007/978-3-662-47854-7_33.

[120] Sompolinsky, Yonatan, Yoad Lewenberg, and Aviv Zohar. "SPECTRE: a fast and scalable cryptocurrency protocol." IACR Cryptol. ePrint Arch. 2016, no. 1159 (2016).

[121] Sompolinsky, Yonatan, and Aviv Zohar. "Phantom." IACR Cryptology ePrint Archive, Report 2018/104 (2018). 
[122] Li, Chenxing, Peilun Li, Dong Zhou, Wei Xu, Fan Long, and Andrew Yao. "Scaling nakamoto consensus to thousands of transactions per second." arXiv preprint arXiv:1805.03870 (2018).

[123] "DagCoin: Whitepaper: a cryptocurrency without blocks", https://bitslog.files.wordpress.com/2015/09/dagcoin-v41.pdf (accessed on 05 May 2021).

[124] Teutsch, Jason, and Christian Reitwießner. "A scalable verification solution for blockchains." arXiv preprint arXiv:1908.04756 (2019).

[125] Kalodner, Harry, Steven Goldfeder, Xiaoqi Chen, S. Matthew Weinberg, and Edward W. Felten. "Arbitrum: Scalable, private smart contracts." In 27th (USENIX) Security Symposium (USENIX, Security 18), (2018):1353-1370.

[126] Wood, G. (2016). Polkadot: Vision for a heterogeneous multi-chain framework. White Paper. Available online: https://www.win.tue.nl/ mholende/seminar/references/ethereum_polkadot.pdf (accessed on 05 May 2021). 